\title{
Large-Eddy Simulation of Contrails
}

\author{
ANDREAS CHLOND \\ Max-Planck-Institut für Meteorologie, Hamburg, Germany
}

(Manuscript received 17 July 1996, in final form 8 July 1997)

\begin{abstract}
Numerical simulations of contrails have been performed to investigate the role of various external parameters and physical processes in the life cycle of contrails. The general idea underlying the model is that of a largeeddy model. The model explicitly represents the large-scale three-dimensional motions (10-m grid resolution), while small-scale turbulence is parameterized; it contains a detailed microphysical model and it takes into account infrared radiative cooling in cloudy conditions and the vertical shear of the ambient flow.

The model is applied to conditions typical for those under which contrails could be observed, that is, in an atmosphere which is supersaturated with respect to ice and at a temperature of $220 \mathrm{~K}$. The simulations begin in the late dispersion phase (i.e., about $10^{3} \mathrm{~s}$ after exhaust) and trace the evolution of the contrails for a halfhour period. Coherent structures can be identified within these clouds with vertical velocity fluctuations of the order of $0.1 \mathrm{~m} \mathrm{~s}^{-1}$ that are generated mainly by buoyancy due to latent heat release. In addition, the sensitivity runs undertaken as a test of the model to a change in significant physical processes or external parameters indicate that the contrail evolution is controled primarily by humidity, temperature, and static stability of the ambient air and secondarily by the baroclinicity of the atmosphere. Moreover, it turns out that the initial ice particle concentration and radiative processes are of minor importance in the evolution of contrails, at least during the 30-min simulation period.
\end{abstract}

\section{Introduction}

During the last decade growing debate about the role of anthropogenic activities in climate and climate change have also given rise to increasing concern about the possible effects of global air traffic on climate (e.g., Grassl 1990; Johnson et al. 1992). Although the absolute emissions by air traffic (a few percent of the total) are small compared to surface emissions, their effects are potentially important, as the emissions from aircraft are the dominant source for pollution in the upper troposphere/lower stratosphere (Schumann 1994).

In terms of atmospheric impact of aviation there are two key areas of concern. The first focuses on chemical effects and mainly on how the injection of nitrogen oxides $\left(\mathrm{NO}_{x}\right)$ by aircraft at cruising altitudes may influence the atmospheric ozone chemistry (see, e.g., Schumann 1995 and references therein). The second deals with the impact on the radiation budget of the atmosphere through contrail formation. Contrails are initiated in the jet region, that is, in the near-exhaust trail of a jet aircraft, when the hot water vapor in the exhaust gases is mixed with moist environmental air. According to Appleman (1953) and Schumann (1996),

Corresponding author address: Dr. Andreas Chlond, Max-PlanckInstitut für Meteorologie, Bundesstrasse 55 D-20146, Hamburg, Germany.

E-mail: chlond@dkrz contrails form when the water-vapor mixing ratio during the mixing process exceeds saturation with respect to water. In this case the water vapor condenses into liquid droplets, which may freeze afterward if the environmental temperature is below $-40^{\circ} \mathrm{C}$. If the atmosphere is supersaturated with respect to ice, contrails can persist for a long time and can trigger additional ice clouds (Schumann and Wendling 1990).

It is known that ice clouds enhance backscattering of sunlight as well as thermal emission. The net effect is uncertain because the competition between solar albedo and infrared emission effects involving ice clouds is dependent on such factors as cloud position, cloud optical thickness, and microphysical properties. Contrails are expected to enhance the "greenhouse effect," owing to their low optical thickness (Sassen 1991; Sassen et al. 1989; Stephens and Webster 1981; Stephens et al. 1990).

The climatic impact of contrails and of cirrus clouds that develop from contrails is due to their optical characteristics and to their areal coverage. With respect to additional cloud cover due to contrails, the estimates are very uncertain. Analysis of satellite data for a period of a few years duration over Europe and the eastern part of the North Atlantic indicate maximum values along the transatlantic flight corridor around $50^{\circ} \mathrm{N}$ (of almost $2 \%$ ) and over western Europe resulting in $0.5 \%$ contrail cloudiness on the average (Bakan et al. 1994). The effect of contrails on climate has been studied by means of 
one-dimensional models (e.g., Liou 1992; Fortuin et al. 1995) and by means of a three-dimensional general circulation model (Ponater et al. 1996). These studies indicate that contrails may have a significant effect on the planet's radiation balance and hence on climate. However, the effect of changes in thin cirrus cloud cover results in a surface temperature response pattern whose magnitude is significant only for a mean contrail coverage exceeding present-day amounts (Ponater et al. 1996).

In situ information about persistent contrails has been provided by Knollenberg (1972) and Gayet et al. (1996). These studies demonstrate that large contrails could frequently be observed in the atmosphere and that the total ice mass of such contrails can be much larger than to be expected from the amount of water emitted by the aircraft. Hence, the hypothesis exists that large, persistent contrails form by triggering ice formation in ambient air that is subsaturated with respect to water but supersaturated with respect to the ice phase (Detwiler and Pratt 1984). Therefore, it might be useful to apply a numerical model to study the contrail evolution and to test this hypothesis.

Only a few models of contrail evolution have been developed, each focusing on particular physical processes such as ice nucleation, radiative forcing, or cloudscale motions, while parameterizing other processes. Boin and Levkov (1994a,b) studied the influence of ambient humidity on the lifetime of contrails and also investigated the sensitivity of contrail evolution with respect to various parameterizations of ice nucleation in this model. They found a nearly exponential increase of lifetime with increasing humidity. Gierens (1996) performed numerical simulations of persistent contrails using a two-dimensional model that includes dynamical, radiative, and microphysical processes. The microphysical processes were parameterized in terms of measurable quantities, such as water content. He found that the spreading of contrails is mainly caused by the secondary and higher-order vortices that develop as a reaction of the atmosphere on the downward traveling pair of primary vortices, which in turn are produced by the aircraft itself.

Here we have developed a large-eddy simulation model of contrail evolution including microphysical, dynamical, and radiative processes. Unlike earlier contrail models, which were subject to limitations in the ability to resolve the actual eddy structure, our primary interest is in simulating the temporal development of three-dimensional secondary flows within contrails under conditions where latent heat release and radiative fluxes should have a strong effect. Hence, our study confines to the simulation of contrails in the late socalled dispersion regime, that is, about $10^{3} \mathrm{~s}$ after the passage of the aircraft, because only in this phase large, persistent contrails can evolve, which in turn may have an impact on climate. During this phase we assume that the evolution of the contrail is controlled by the inter- action of the ice cloud with the background turbulence field (Dürbeck and Gerz 1995) and by self-induced dynamics due to differential radiative heating and due to latent heat release (see section 4). The general idea underlying the model is that of a large-eddy model. The model explicitly represents large-scale three-dimensional motions (10-m grid resolution), while small-scale turbulence is parameterized. The model includes most of the physical processes occurring in ice clouds in the absence of solar radiation. It contains a treatment of the subgrid-scale turbulence that incorporates the effects of thermal stratification; it takes into account infrared radiative cooling in cloudy conditions (using an effective emissivity model) and the influence of vertical shear of the ambient flow. Moreover, we explicitly resolve the size distribution of ice particles and calculate the evolution of the size distributions including processes such as deposition growth, transport, and gravitational settling. The model is applied to conditions typical for those under which persistent contrails could be observed, that is, in an atmosphere which is approximately saturated with respect to ice and at a temperature of approximately $230 \mathrm{~K}$ or colder. The simulations start at the beginning of the dispersion phase and trace the evolution of the contrails for a half-hour duration. The objectives of the present study are first to investigate the secondary flow structure within persistent contrails and to understand the processes that cause contrails to spread out to form cirrus clouds. Second, we want to examine the respective roles of external conditions, such as temperature, humidity, static stability, and baroclinicity of the ambient atmosphere in the evolution of contrails. Finally, the influence of the initial ice particle concentration and the importance of physical processes such as vapor deposition and radiation for the life cycle on contrails is examined.

This paper is organized as follows. In section 2, the large-eddy simulation model is introduced. The design of the numerical simulation is described in section 3 . The simulation results are presented and discussed in section 4. Finally, a summary and conclusions are given in section 5 .

\section{The model}

To study the evolution of contrails, we have developed a three-dimensional, time-dependent, Eulerian model that has three coupled components: a basic dynamical model, a cloud microphysical model, and a radiative transfer model. These components and some numerical computational issues are described in this section.

\section{a. The basic dynamic model}

The basic dynamical framework employed is a modified and upgraded version of a large-eddy simulation (LES) model, which has been described in Chlond (1992). The model explicitly calculates the large (re- 
solved) scale motions, while the small (subgrid) scale motions have to be parameterized. The equations governing the resolved-scale motions are the three-dimensional, spatially averaged, incompressible Boussinesq equations conserving mass and momentum. The Bous- sinesq approximation has been applied, which is appropriate since significant convective motions within contrails will be limited to a shallow layer $(\sim 1 \mathrm{~km})$ within the elevated computational domain.

In Cartesian coordinates the equations are

$$
\begin{aligned}
\frac{\partial \bar{u}}{\partial x}+\frac{\partial \bar{v}}{\partial y}+\frac{\partial \bar{w}}{\partial z} & =0 \\
\frac{\partial \bar{u}}{\partial t} & =-\frac{\partial}{\partial x}(\bar{u} \bar{u})-\frac{\partial}{\partial y}(\bar{v} u)-\frac{\partial}{\partial z}(\bar{w} \bar{u})-\frac{1}{\rho_{00}} \frac{\partial \bar{p}}{\partial x}+f\left(v-v_{g}\right)-\frac{\partial}{\partial x} \tau_{11}-\frac{\partial}{\partial y} \tau_{12}-\frac{\partial}{\partial z} \tau_{13} \\
\frac{\partial \bar{v}}{\partial t} & =-\frac{\partial}{\partial x}(\bar{u} \bar{v})-\frac{\partial}{\partial y}(\bar{v} \bar{v})-\frac{\partial}{\partial z}(\bar{w} \bar{v})-\frac{1}{\rho_{00}} \frac{\partial \bar{p}}{\partial y}-f\left(u-u_{g}\right)-\frac{\partial}{\partial x} \tau_{21}-\frac{\partial}{\partial y} \tau_{22}-\frac{\partial}{\partial z} \tau_{23} \\
\frac{\partial \bar{w}}{\partial t} & =-\frac{\partial}{\partial x}(\bar{u} \bar{w})-\frac{\partial}{\partial y}(\bar{v} \bar{w})-\frac{\partial}{\partial z}(\bar{w} \bar{w})-\frac{1}{\rho_{00}} \frac{\partial \bar{p}}{\partial z}+\frac{g}{\Theta_{00}}\left(\bar{\Theta}_{v}-\left\langle\bar{\Theta}_{v}\right\rangle\right)-\frac{\partial}{\partial x} \tau_{31}-\frac{\partial}{\partial y} \tau_{32}-\frac{\partial}{\partial z} \tau_{33},
\end{aligned}
$$

where overbars signify the Reynolds average over the grid volume $\Delta x \cdot \Delta y \cdot \Delta z$, and the horizontal average is denoted by the angular brackets. Here, $u, v$, and $w$ are the velocity components; $p$ is the perturbation pressure; $f$ is the Coriolis parameter; $g / \Theta_{00}$ is the buoyancy parameter; and $\rho_{00}$ is the reference density, which is assumed to be independent of height. The components of the geostrophic wind $u_{g}$ and $v_{g}$ are fixed in time but can depend on altitude, and $\tau_{i j}$ is the subgrid stress tensor given by

$$
\tau_{i j}=-\left(\overline{u_{i} u_{j}}-\bar{u}_{i} \bar{u}_{j}\right)+\frac{1}{3}\left(\overline{u_{k}^{2}}-\bar{u}_{k}^{2}\right) \delta_{i j},
$$

where $\delta_{i j}$ is the Kronecker delta.

In order to describe the thermodynamic properties of the cloudy atmosphere, the thermodynamics is represented by the conservation equation for the ice-liquid water potential temperature $\Theta_{i}$ and the total water content $q=q_{v}+q_{i}$, where $q_{v}$ is the specific humidity and $q_{i}$ the ice content. These equations are given by

$$
\begin{aligned}
& \frac{\partial \bar{\Theta}_{i}}{\partial t}=-\frac{\partial}{\partial x}\left(\bar{u} \bar{\Theta}_{i}\right)-\frac{\partial}{\partial y}\left(\bar{v} \bar{\Theta}_{i}\right)-\frac{\partial}{\partial z}\left(\bar{w} \bar{\Theta}_{i}\right)-\frac{\partial}{\partial x} H_{1}-\frac{\partial}{\partial y} H_{2}-\frac{\partial}{\partial z} H_{3}+\left(\frac{\partial \bar{\Theta}_{i}}{\partial t}\right)_{\mathrm{RAD}}+\left(\frac{\partial \bar{\Theta}_{i}}{\partial t}\right)_{\mathrm{SED}}, \\
& \frac{\partial \bar{q}}{\partial t}=-\frac{\partial}{\partial x}(\bar{u} \bar{q})-\frac{\partial}{\partial y}(\bar{v} \bar{q})-\frac{\partial}{\partial z}(\bar{w} \bar{q})-\frac{\partial}{\partial x} Q_{1}-\frac{\partial}{\partial y} Q_{2}-\frac{\partial}{\partial z} Q_{3}+\left(\frac{\partial \bar{q}}{\partial t}\right)_{\mathrm{SED}},
\end{aligned}
$$

where $\left(\partial \bar{\Theta}_{i} / \partial t\right)_{\text {RAD }}$ is a cooling term simulating radiative cooling; $\left(\partial \bar{\Theta}_{i} / \partial t\right)_{\text {SED }}$ and $(\partial \bar{q} / \partial t)_{\text {SED }}$ describe the effect of sedimentation of ice particles on the variables $\Theta_{i}$ and $q$, respectively. These terms are derived from parameterizations discussed in sections $2 \mathrm{~b}$ and $2 \mathrm{c}$, respectively; $H_{i}$ is the turbulent heat flux and $Q_{i}$ represents the turbulent total moisture flux given by

$$
\begin{aligned}
H_{i} & =-\overline{u_{i} \Theta_{i}}+\bar{u}_{i} \bar{\Theta}_{i} \\
Q_{i} & =-\overline{u_{i} q}+\bar{u}_{i} \bar{q}_{i} .
\end{aligned}
$$

The ice-liquid water potential temperature $\Theta_{i}$, suggested by Tripoli and Cotton (1981), is defined by

$$
\Theta_{i}=\Theta-\left(\frac{\Theta}{T}\right) \frac{L}{c_{p}} q_{i},
$$

where $\Theta$ is the potential temperature, $T$ is the absolute temperature, $L$ is the latent heat coefficient of sublimation, and $c_{p}$ represents the specific heat of dry air. We further assume that

$$
\frac{\Theta}{T}=\left(\frac{p_{00}(0)}{p_{00}(z)}\right)^{0.286},
$$

where $\mathrm{p}_{00}$ denotes the hydrostatic reference pressure.

In the buoyancy term, $\Theta_{v}$ is the virtual potential temperature defined by 


$$
\Theta_{v}=\Theta\left(1+0.61 q-1.61 q_{i}\right) .
$$

The ice content $q_{i}$ is calculated using the microphysical formulation described in section $2 b$.

The spatially averaged equations discussed above can be solved once the subgrid-scale (SGS) fluxes are determined in terms of the resolved-scale fields. Our subgrid flux model is based on a transport equation for the SGS kinetic energy,

$$
\bar{e} \equiv \frac{1}{2}\left(\overline{u_{k}^{2}}-\bar{u}_{k}^{2}\right)
$$

and is similar to that of Deardorff (1980). We write the SGS equation as

$$
\begin{aligned}
\frac{\partial \bar{e}}{\partial t}+\frac{\partial}{\partial x_{j}}\left(\bar{u}_{j} \bar{e}\right)= & -\tau_{i j} \frac{\partial \bar{u}_{i}}{\partial x_{j}}+\frac{g}{\Theta_{00}} \overline{u_{3}^{\prime} \Theta_{v}} \\
& -\frac{\partial}{\partial x_{j}}\left(\overline{u_{j}^{\prime} e^{\prime}}+\overline{u_{j}^{\prime} \frac{p^{\prime}}{\rho_{00}}}\right)-c_{\varepsilon} \frac{e^{3 / 2}}{\Lambda}
\end{aligned}
$$

where the first two terms on the right-hand side are the production terms due to local shear and buoyancy, respectively. The third term is a transport term and the last term is the viscous dissipation; $\Lambda$ is a length scale and $c_{\varepsilon}$ is a stability-dependent coefficient; both are specified algebraically below. The subgrid fluxes were parameterized according to first-order closure by

$$
\begin{aligned}
\tau_{i j} & =-K_{M}\left(\frac{\partial \bar{u}_{i}}{\partial x_{j}}+\frac{\partial \bar{u}_{j}}{\partial x_{i}}\right) \\
H_{i} & =-K_{H} \frac{\partial \bar{\Theta}_{i}}{\partial x_{i}} \\
Q_{i} & =-K_{H} \frac{\partial \bar{q}}{\partial x_{i}} \\
\overline{u_{i}^{\prime} e^{\prime}}+\overline{u_{i}^{\prime} \frac{p^{\prime}}{\rho_{00}}} & =-K_{H} \frac{\partial \bar{e}}{\partial x_{i}} \\
\frac{u_{3}^{\prime} \Theta_{v}^{\prime}}{2} & =K_{1} \overline{u_{3}^{\prime} \Theta(}+K_{2} \overline{u_{3}^{\prime} q^{\prime}},
\end{aligned}
$$

where $K_{M}$ is a subgrid-scale eddy coefficient for momentum and $K_{H}$ is a subgrid-scale eddy coefficient for scalar quantities, which are given by

$$
K_{1}=1-\frac{r \alpha\left[\frac{L}{c_{P}}-1.61 \Theta_{00}\left(\frac{\Theta}{T}\right)^{-1}\right]}{1+\alpha \frac{L}{c_{p}}},
$$

where

$$
\alpha \equiv\left(\frac{\partial q_{s}}{\partial T}\right)_{T=\bar{T}_{i}}=0.622 L q_{s}\left(\bar{T}_{i}\right) /\left(R_{d} \bar{T}_{i}^{2}\right) .
$$

Here $R_{d}$ is the gas constant for dry air; $\bar{T}_{i}$ is the iceliquid water temperature

$$
\overline{\mathrm{T}}_{i}=\left(\frac{\Theta}{T}\right)^{-1} \bar{\Theta}_{i},
$$

and $q_{s}$ is the saturation specific humidity, which is obtained from the relation

$$
q_{s}(T, p)=0.622 e_{s}(T) /\left(p-0.378 e_{s}(T)\right) .
$$

The saturation vapor pressure with respect to a plane surface of pure ice is determined according to

$$
\ln e_{s}=-6147.795166 / T+24.31720686,
$$

where $e_{s}$ is in $10^{2} \mathrm{~Pa}$ (Laube and Höller 1986). The expressions (16a)-(16b) are simplified versions of those derived in Sommeria and Deardorff (1977), where we have neglected $q_{s}$ in comparison with unity and we used the reference temperature $\Theta_{00}$ in place of $\bar{\Theta}_{i}$. Here $r$ denotes the subgrid-scale cloud fraction, which is either zero $\left(q_{i}=0\right)$ or unity $\left(q_{i}>0\right)$.

The eddy coefficients are proportional to the product of a length scale and the subgrid turbulent kinetic energy. Accordingly we have

$$
\begin{aligned}
& K_{M}=c_{M} \Lambda e^{1 / 2} \\
& K_{H}=c_{H} \Lambda e^{1 / 2},
\end{aligned}
$$

where, according to Deardorff (1980), $c_{M}$ and $c_{H}$ are given by

$$
\begin{aligned}
& c_{M}= 0.1 \\
& c_{H}= \begin{cases}3 c_{M}, & R_{i} \leq 0 \\
\left(1+\frac{2 \Lambda}{\min (0.7 z, \Delta)}\right) c_{M}, & R_{i}>0 .\end{cases}
\end{aligned}
$$

The turbulent Richardson number $\mathrm{Ri}$ is defined as

$$
\mathrm{Ri}=\left(\frac{g}{\Theta_{00}}\right)\left(K_{1} \frac{\partial \bar{\Theta}_{i}}{\partial z}+K_{2} \frac{\partial \bar{q}}{\partial z}\right) \frac{\Lambda^{2}}{2 e},
$$

where the length scale $\Lambda$ is given by

$$
\Lambda=\min \left(0.7 z, \Delta, 0.76 e^{1 / 2} N^{-1}\right) .
$$

Here $\Delta$ is the average grid scale $\Delta=(\Delta x+\Delta y+\Delta z) / 3$, where $\Delta x, \Delta y$, and $\Delta z$ are the respective grid intervals and $N$ is the Brunt-Väisälä frequency, defined by

$$
N^{2}=g / \Theta_{00}\left(K_{1} \frac{\partial \bar{\Theta}_{i}}{\partial z}+K_{2} \frac{\partial \bar{q}}{\partial z}\right) .
$$

Hence, $\Lambda$ is proportional to $z$ near the surface but is limited to the average mesh spacing. The third limit is applied only in stable region where $N^{2}>0$. Following 
Deardorff (1980), the constant in the dissipation term is calculated as

$$
c_{\varepsilon}= \begin{cases}0.7, & \mathrm{Ri} \leq 0 \\ \left(0.19+0.51 \frac{\Lambda}{\min (0.7 z, \Delta)}\right), & \mathrm{Ri}>0 .\end{cases}
$$

\section{b. The cloud microphysical model}

To describe cloud physical processes a model with detailed treatment of microphysical processes is used. As a first approximation the ice particles are treated as spherical frozen drops. In this case, the ice particle spectrum could be represented by a one-dimensional number density function $f(r, \mathbf{x}, t)$. Following the density function notation of Berry (1967), this function is defined such that $f(r, \mathbf{x}, t) \mathrm{dr}$ is the number of ice particles per unit mass between the radius $r$ and $r+d r$ at the location $\mathbf{x}=(x, y, z)$ and at time $t$. The analytical form of the equation governing the time rate of change of the number density function is written as

$$
\begin{aligned}
\frac{\partial f}{\partial t} & +\frac{\partial}{\partial x_{i}}\left(u_{i} f\right)+\frac{\partial}{\partial z}\left(w_{T} f\right)+\frac{\partial}{\partial r}\left(\frac{d r}{d t} f\right) \\
+ & \frac{\partial}{\partial x_{i}}\left(K_{H} \frac{\partial}{\partial x_{i}} f\right)=0 .
\end{aligned}
$$

The second and the third term on the left-hand side of Eq. (23) represent advection of the ice particles with the air's velocity and sedimentation of particles with their terminal velocity $w_{T}$. Values for $\mathrm{w}_{\mathrm{T}}$ are calculated following Beard (1976). The fourth term on the lefthand side of Eq. (23) represents the spectral spreading and shifting effect on $f$ due to vapor deposition/sublimation where $d r / d t$ is the Lagrangian diffusional growth rate of a particle of radius $r$. The last term represents turbulent particle mixing. Equation (23) reveals that collision-aggregation processes and nucleation processes are not included in the model. The reason for the first assumption is that aggregation is of minor importance at temperatures below $-20^{\circ} \mathrm{C}$, presumably because ice crystals do not stick together when they collide at these temperatures (Hobbs et al. 1974). Admittedly, we must acknowledge that, in light of more recent results and observations, aggregation may be more significant than previously thought (Kajikawa and Heymsfield 1989; Mitchell 1988, 1991, 1994). However, we believe that ignoring this process in the present work is likely not of great consequence. The justification for the latter assumption appears more serious because it seems unlikely that no new cloud particles are allowed to form in a supersaturated atmosphere, especially in the presence of dynamical disturbances such as contrails. However, we think that due to the fact that the aircraft emits countless $\mathrm{CCN}$ in the air along with water vapor to produce a contrail containing a high concentration of ice particles, the most important cloud physical processes in contrails are diffusional growth and sublimation of ice particles; hence nucleation of new ice particles should be of secondary importance.

The rate of growth of ice particles is determined by considering the simultaneous transport of mass and energy from a falling ice particle. The equation governing the rate of growth by condensation is similar to that of Young (1974) except that the terms for the change in particle temperature with time and the effects of accretion of water drops on an ice particle are neglected. The governing equation for diffusional growth rate of a spherical ice particle of radius $r$ that includes radiative heat exchange between the ice particle and its environment is

$$
r \frac{d r}{d t}=\frac{1}{C_{1}}\left[C_{2}(S-1)-\frac{\Delta F}{4 \pi r}\right],
$$

where

$$
\begin{aligned}
C_{1} & =L \rho_{i}+\frac{R_{v} T \rho_{i}}{D^{\prime} e_{s}(T)} \cdot C_{2}, \\
C_{2} & =\frac{\lambda^{\prime} R_{v} T^{2}}{L} .
\end{aligned}
$$

Here $R_{v}$ is the gas constant for water vapor and $\rho_{i}$ is the density of ice. The quantity $\lambda^{\prime}$ denotes the thermal conductivity of moist air and $D^{\prime}$ the diffusivity of water vapor, where both terms are modified to take into account gas kinetic and ventilation effects on the diffusional growth following Hall and Pruppacher (1976). The first part of the right-hand side of Eq. (24) describes the usual particle growth equation without the radiation term. The quantity $S$ is the ratio of the actual specific humidity of the ambient air $q_{v}$ and its saturation value $q_{s}(T, p)$ :

$$
S=\frac{q_{v}}{q_{s}(T, p)} .
$$

The second part of Eq. (24) describes the impact of radiative heat transfer on the particle growth. Here $\Delta F$ is the net longwave radiational energy gain at the particle surface. Following Bott et al. (1990), this quantity could be written as

$$
\Delta F=\pi r^{2} Q_{\mathrm{abs}}(r)\left[2\left(F^{+}+F^{-}\right)-4 B(T)\right],
$$

where $B, F^{+}$, and $F^{-}$denote the Planck function and the upward and downward broadband longwave radiative fluxes, respectively; $Q_{\text {abs }}(r)$ is the efficiency factor for absorption, assumed to be unity for simplicity.

The ice content $q_{i}$ is obtained by integrating the product of the number density function $f(r, \mathbf{x}, t)$ and the mass of a single ice particle $m_{i}(s)$ over the entire particle spectrum:

$$
q_{i}(x, t)=\int_{0}^{\infty} m_{i}(r) f(r, \mathbf{x}, t) d r .
$$


The terms $(\partial \bar{q} / \partial z)_{\mathrm{SED}}$ and $\left(\partial \bar{\Theta}_{i} / \partial t\right)_{\mathrm{SED}}$ in Eqs. (6) and (7), which describe the effect of sedimentation on the variables $q$ and $\Theta_{i}$, can be obtained as follows:

$$
\begin{aligned}
& \left(\frac{\partial \bar{q}}{\partial t}\right)_{\mathrm{SED}}=-\frac{\partial}{\partial z} \int_{0}^{\infty} w_{T}(r) m_{i}(r) f(r, \mathbf{x}, t) d r \\
& \left(\frac{\partial \bar{\Theta}_{i}}{\partial t}\right)_{\mathrm{SED}}=-\left(\frac{\Theta}{T}\right) \frac{L}{c_{p}}\left(\frac{\partial \bar{q}}{\partial t}\right)_{\mathrm{SED}} .
\end{aligned}
$$

\section{c. Radiative transfer model}

The radiative transfer model is used to calculate radiative cooling rates for use in the thermodynamic equation and in the ice particle condensational growth term. We utilize a simple radiative transfer parameterization scheme for application to the LES cloud model, which only takes into account infrared absorption and emission by ice particles. Radiative cooling due to gaseous absorbers and solar radiative heating are excluded. The longwave scheme is based on the parameterization of cloud "effective emissivity" (Cox 1976). This quantity allows the calculation of broadband infrared fluxes and cooling rates within the cloud. For the flux computations at a given level $z$, the atmosphere is supposed to be isothermal above and below $z$. Vertical columns are considered independent and any lateral effect is neglected. Upward and downward radiative fluxes $F^{+}$and $F^{-}$are written

$$
\begin{aligned}
& F^{+}(z)=F^{+}\left(z_{B}\right)+\varepsilon^{+}\left(z, z_{B}\right)\left(B(T(z))-F^{+}\left(z_{B}\right)\right) \\
& F^{-}(z)=F^{-}\left(z_{T}\right)+\varepsilon^{-}\left(z, z_{T}\right)\left(B(T(z))-F^{-}\left(z_{T}\right)\right),
\end{aligned}
$$

where $B(T(z))$ is the blackbody emission at the temperature of the level $z ; F^{+}\left(z_{B}\right)$ and $F^{-}\left(z_{T}\right)$ are the impinging fluxes at the bottom and at the top of the model domain, respectively, which have to be prescribed. Here $\varepsilon^{+}\left(z, z_{B}\right)$ and $\varepsilon^{-}\left(z, z_{T}\right)$ are the effective emissivities of ice between the bottom of the model domain $\left(z=z_{B}\right)$, and between level $z$ and the top of the model domain $\left(z=z_{T}\right)$, respectively. They are given by

$$
\begin{aligned}
& \varepsilon^{+}\left(z, z_{B}\right)=1-\exp \left(-a_{0}^{+} \operatorname{IWP}\left(z_{B}, z\right)\right), \\
& \varepsilon^{-}\left(z, z_{T}\right)=1-\exp \left(-a_{0}^{-} \operatorname{IWP}\left(z, z_{T}\right)\right),
\end{aligned}
$$

where $\operatorname{IWP}\left(z_{B}, z\right)$ and $\operatorname{IWP}\left(z, z_{T}\right)$ are the amounts of ice contained in the columns below and above level $z$. From Eqs. (29a) and (29b), $a_{0}$ defines a mass absorption coefficient $\left(\mathrm{m}^{2} \mathrm{~kg}^{-1}\right)$, which is given by Starr and Cox (1985) as

$$
a_{0}^{+}=70 a_{0}^{-}=50 .
$$

The radiation computation is made at each time step for each column of air. The source term in Eq. (6) can now be written as

$$
\left(\frac{\partial \bar{\Theta}_{i}}{\partial t}\right)_{\mathrm{RAD}}=-\left(\frac{\Theta}{T}\right) \frac{1}{\rho_{00} c_{p} \Delta z}\left(\Delta F\left(z^{+}\right)-\Delta F\left(z^{-}\right)\right),
$$

where $\Delta \mathrm{F}$ is the difference between upward and downward fluxes at two consecutive levels around the level where $\bar{\Theta}_{i}$ is defined.

\section{d. Computational grid, boundary conditions, and numerical techniques}

Since finite difference schemes suffer from numerical errors, it is particularly important to study which grid resolution and domain size are adequate to give a reliable representation of the phenomenon under consideration. Therefore, we carried out two-dimensional test simulations in a domain of size $640 \times 640 \mathrm{~m}^{2}$ with grid spacings of $\Delta x=\Delta z=20 \mathrm{~m}, \Delta x=\Delta z=13.3 \mathrm{~m}, \Delta x$ $=\Delta z=10 \mathrm{~m}$, and $\Delta x=\Delta z=5 \mathrm{~m}$. It was found that the solution converges in terms of the vertical velocity variance, integrated ice content, and diffusion parameters, and that the resolution $\Delta x=\Delta z=10 \mathrm{~m}$ is sufficient. Moreover, it turns out that the circulations induced by the contrail often span at the end of the simulations a great portion of the model domain, so the width of the domain may be having an effect on the results. To demonstrate that this has little effect on contrail evolution we also performed a simulation with a domain $1280 \mathrm{~m}$ wide instead of $640 \mathrm{~m}$ while maintaining the grid resolution. It appears that the restricted domain size does not affect significantly the evolution of the contrail plume in the control case. The results in the extended domain are almost identical to those of the restricted domain.

Therefore, the spatial domain is specified as an elevated box that extends horizontally and vertically over a finite domain of size $640 \times 640 \times 640 \mathrm{~m}^{3}$. The domain is divided into $64 \times 64 \times 64$ grid boxes (i.e., $\Delta x=$ $\Delta y=\Delta z=10 \mathrm{~m}$ ). At the lateral boundaries, periodicity is assumed. At the bottom and top, a zero slope condition on all variables except $\bar{w}, \bar{q}$, and $\bar{\Theta}_{i}$, is imposed. Vertical velocity $\bar{w}$ is zero at the bottom and the top and the gradients of $\bar{q}$ and $\bar{\Theta}_{i}$ are fixed at the initial gradients $\Gamma_{q}$ and $\Gamma_{\Theta_{i}}$. Although $\partial f / \partial z=0$ is formally maintained at the lower boundary, cloud ice is permitted to exist across the lower boundary by virtue of its relative fall speed. These boundary conditions may be unrealistic if the region near the lower or upper boundaries becomes disturbed. This is prevented by on-time termination of the simulation. Furthermore, at the lower and upper right levels of the model there are sponge layers that remove fluctuations of the velocity, temperature, and humidity fields in order to damp disturbances there.

The numerical integration scheme is based on an equidistant staggered grid and finite difference approximations. All scalars are defined at the cell centers, $\bar{u}$ is evaluated at the middle of the upstream and downstream faces, $\bar{v}$ at the middle of the two cross-stream faces, and $\bar{w}$ at the middle of the bottom and top faces.

The advection terms of the momentum equations are treated by a scheme proposed by Piacsek and Williams 
(1970). It is a second-order method in space, which conserves the integral of linear and quadratic quantities up to very small errors. The diffusion terms are solved with a second-order, spatial central difference scheme. Time integration of the momentum equation is performed using the Adams-Bashforth scheme. The Poisson equation for the pressure is solved using a fast Fourier (in the horizontal directions) and a finite-difference technique (in the vertical direction).

The integration method used to solve Eqs. (6)-(7) and (13) is based on a fully explicit, time-splitting method. Terms representing the divergence of the vertical flux due to advection and sedimentation are treated by the monotone, locally modified version of Bott's advection scheme (Chlond 1994). All other terms are advanced with the Euler method in time and are resolved with the second-order central-difference scheme in space.

The numerical treatment of the ice particle continuity equation (23) is similar to that of Eqs. (6)-(7) and (13). However, special care has to be taken in the handling of the diffusional growth of ice particles. As pointed out in section $2 \mathrm{~b}$, ice particles are represented in terms of a one-dimensional distribution function with the radius of the particle as the independent variable.

For numerical purposes the ice particle size distribution is subdivided into 12 classes with equidistant grid spacing in logarithmic scales, resulting in a radius grid that spans from $r_{\min }=1 \mu \mathrm{m}$ to $r_{\max }=64 \mu \mathrm{m}$. As can be seen from the fourth term of Eq. (23), the diffusional growth may be formulated as a flux divergence in radius space due to condensational growth and sublimation of ice. The numerical procedure to treat this process is a one-dimensional application of the locally modified version of Bott's advection scheme. However, since the particle growth velocity depends on the supersaturation, it is a function of time. Arnason and Brown (1971) have shown that due to this time dependency the use of an explicit method to integrate this equation is not suitable since it requires extremely small time steps for numerical stability. In order to allow larger integration time steps, the condensational growth process is implicitly solved similar to the method of Hall (1980) and Bott et al. (1990). At time step $n$ the diffusional growth of the particles is calculated with a constant value of the saturation value $\bar{S}=\left(S^{n}+S_{0}^{n+1}\right) / 2$, where $S^{n}$ is the actual saturation value and $S_{0}^{n+1}$ is a first guess (e.g., $\left.S_{0}^{n+1}=1\right)$. Utilizing Newton-Raphson method, the final value $S_{m}^{n+1}$ is iteratively determined by requiring $\left|S_{m}^{n+1}-S_{m-1}^{n+1}\right|<10^{-6}$.

A time step of $1.5 \mathrm{~s}$ was used for all runs. Each 30min simulation required about 12 hours of CPU time on one processor of a CRAY C916 computer.

\section{Design of numerical experiments}

The primary goal of this study is to demonstrate the capabilities of the model and to illustrate, under simplified atmospheric conditions, the basic physical mechanisms of contrail development. Since we are interested in persistent contrails, which may have climatic impact, our study concentrates on the simulation of contrails in the late dispersion phase, that is, about $10^{3} \mathrm{~s}$ after exhaust. During this phase the evolution of the contrail is controlled by the interaction of the ice cloud with the background turbulence field and by self-induced dynamics due to differential radiative heating and due to latent heat release. Therefore, to avoid unnecessary complications at this stage, the contrail evolution is studied in an atmospheric environment characterized by horizontally homogeneous stratifications of temperature, humidity, and momentum.

To set up the control run, which serves as a reference case, we have to define the geographical latitude, set the external parameters that characterize the large-scale fields, and specify the initial conditions for all prognostic variables. The parameters and initial conditions for the model have been chosen to reproduce more or less the conditions met during which persistent contrails could be observed in the atmosphere (Schumann 1994; Gayet et al. 1996). We anticipate an air volume at a height of about $10500 \mathrm{~m}$ (i.e., $\mathrm{P}_{00}=250 \mathrm{hPa}$ ) at a reference temperature of $T_{00}=220 \mathrm{~K}$, which is subsaturated with respect to water, that is, $r_{w}=78 \%$ (this corresponds to a supersaturation with respect to ice of $r_{i}=129 \%$ ). Admittedly, a value of the relative humidity with respect to ice as large as $129 \%$ may not be representative of what exists in real world situations because radiosonde profiles even during contrail observations yielded dewpoints at $10 \mathrm{~K}$ lower than the ambient atmosphere. However, it is well known that radiosonde hygrometers could produce systematic error at low temperatures (Pratt 1985). Therefore, we decided to run the model with moderate supersaturation in order to guarantee contrail persistency. The sensitivity of contrail evolution to the imposed humidity field is discussed in more detail in section 4 . The initial potential temperature field was assumed to be horizontal homogeneous within the environment and initially slightly stable with a lapse rate of

$$
\frac{\partial\langle\bar{\Theta}\rangle}{\partial z}=2.5 \times 10^{-3} \mathrm{~K} \mathrm{~m}^{-1} \text {. }
$$

Within the contrail the moist adiabatic temperature gradient was applied. Based on this temperature field, we choose to initialize the moisture field with a relative humidity with respect to ice of $r_{i}^{\text {(env) }}=129 \%$ in the environment of the contrail. Within the contrail we assume that phase relaxation has already occurred, that is, $r_{i}^{\text {(cont) }}=100 \%$. The contrail is specified as two parallel cloud bands in the middle of the model domain elongated in the $y$ direction. The lateral distance between the two cloud cores of the contrail is $50 \mathrm{~m}$. The initial diameter of each of the two cores forming the contrail is $30 \mathrm{~m}$. The initial ice content of the contrail is determined by the amount of water emitted by the aircraft due to the combustion of fuel $\left[15 \mathrm{~g} \mathrm{~m}^{-1}\right.$ for a B747 
TABLE 1. Summary of the numerical simulations.

\begin{tabular}{|c|c|c|c|c|c|c|}
\hline Run & $\begin{array}{l}T_{\mathrm{oo}} \\
(\mathrm{K})\end{array}$ & $\begin{array}{c}\partial \Theta / \partial z \\
(\mathrm{~K} / \mathrm{km})\end{array}$ & $\begin{array}{c}\partial u_{g} / \partial z \\
\left(\mathrm{~s}^{-1}\right)\end{array}$ & $\begin{array}{c}\partial v_{g} / \partial z \\
\left(\mathrm{~s}^{-1}\right)\end{array}$ & $\begin{array}{c}4_{w}\left(r_{i}\right) \\
(\%)\end{array}$ & Remarks \\
\hline 1 & 220 & 0 & 0 & 0 & 78 (129) & - \\
\hline 2 & 210 & 2.5 & 0 & 0 & 85 (153) & - \\
\hline 3 & 220 & 2.5 & 0 & 0 & 78 (129) & control run \\
\hline 4 & 230 & 2.5 & 0 & 0 & 73 (111) & - \\
\hline 5 & 220 & 5 & 0 & 0 & 78 (129) & - \\
\hline 6 & 220 & 2.5 & 0 & $5 \times 10^{-3}$ & 78 (129) & - \\
\hline 7 & 220 & 2.5 & $1 \times 10^{-3}$ & 0 & 78 (129) & - \\
\hline 8 & 220 & 2.5 & 0 & 0 & 66 (109) & - \\
\hline 9 & 220 & 2.5 & 0 & 0 & 90 (149) & - \\
\hline 10 & 220 & 2.5 & 0 & 0 & 78 (129) & $\begin{array}{l}\text { no radiation term in droplet } \\
\text { growth equation }\end{array}$ \\
\hline 11 & 220 & 2.5 & 0 & 0 & 78 (129) & without radiation \\
\hline 12 & 220 & 2.5 & 0 & 0 & 78 (129) & $r_{e}=4.8 \mu \mathrm{m}$ \\
\hline 13 & 220 & 2.5 & 0 & 0 & 78 (129) & $r_{e}=1.7 \mu \mathrm{m}$ \\
\hline
\end{tabular}

aircraft at cruising level; see, e.g., Schumann (1994)] and by the amount of water vapor of environmental air that has been entrained into the contrail and subsequently converted into ice during the jet and the vortex phase, resulting in $\left(\bar{q}_{i}\right)_{\text {cont }}=4.26 \times 10^{-5} \mathrm{~kg} \mathrm{~kg}^{-1}$. Within the contrail the ice particle spectra are initialized as monodisperse distributions, that is, the initial ice mass is concentrated in one bin and the mean effective radius of ice particles was specified as $r_{e}=2.4 \mu \mathrm{m}$, giving a total number of ice particles of $N_{\text {Tот }}=7.87 \times 10^{8} \mathrm{~kg}^{-1}$. The model was run assuming no mean vertical wind shear with the initial condition taken as an atmosphere at rest. The latitude has been taken to be $50^{\circ} \mathrm{N}$ and the impinging longwave radiative fluxes at the bottom and the top of the model are specified as $F^{-}\left(z_{B}\right)=250 \mathrm{~W}$ $\mathrm{m}^{-2}$ and $F^{+}\left(z_{T}\right)=30 \mathrm{~W} \mathrm{~m}^{-2}$, respectively. To initiate convective motions random temperature perturbations of amplitude $10^{-3} \mathrm{~K}$ are imposed at the first time step within the contrail.

Admittedly, the initial conditions chosen for the simulations are highly idealized, which prevent them from being considered as even semirealistic representations of a real world situation. The point is that behind each aircraft a wake is formed that passes through the socalled jet, vortex, and dispersion regime (CIAP 1975). In the vortex phase the wake dynamics is dominated by a vortex pair that results from the rollup of the vortex sheet shed from the aircraft wings. Calculation of Greene (1986) and Louisnard et al. (1995) suggest that-depending on the atmospheric state-10-100 km behind an aircraft the vortex pair has either been totally dissipated by free stream turbulence, buoyancy effects, or translational drag effects or has undergone an instability process and burst so that no coherent vortex of any importance will remain after this distance. However, the real difficulty is that, after the vortices break up, they are gone but their effects are by no means forgotten. Over their lifetime the vortex pair will sink with a typical speed of about $1.5 \mathrm{~m} \mathrm{~s}^{-1}$ (e.g., Gerz and Ehret 1996; Lewellen and Lewellen 1996; Schilling et al. 1996). For a typical commercial aircraft the distance where the trailing vortex pair starts to collapse into turbulence is of order $200 \mathrm{~m}$. At the end of this vortex penetration process, no regular vortex structure can be seen. However, as a dynamical consequence of this process the vortex remnant acquires a temperature and velocity perturbation of order $0.5 \mathrm{~K}$ and $2 \mathrm{~m} \mathrm{~s}^{-1}$ (Gerz and Ehret 1996; Lewellen and Lewellen 1996). These temperature and velocity perturbations are on order of magnitude larger than the maximum temperature and velocity perturbation arising from our simulations (see section 4a). Therefore, since the natural timescale for these motions is a Brunt-Väisälä period (about $10 \mathrm{~min}$ in the present case), the dynamics of contrail evolution considered here is more appropriate for the time period beginning $10^{3} \mathrm{~s}$ after the plane has passed. Keeping this in mind, our simulations should probably be viewed not as a faithful representation of the early timescale of the contrail, but more as a sensitivity study (see below) of ice formation, radiative cooling, and external atmospheric conditions on contrail dynamics.

In addition to the reference case, 12 sensitivity runs have been performed to clarify the influence of various external parameters, initial conditions, and physical processes on the development of contrails (see Table 1). The sensitivity runs 1 to 2 and 4 to 9 (run 3 refers to the control run) were different in that one of the external parameters of the control run is varied while the others are fixed. In this way, we investigate the influence of ambient temperature $T_{00}$ (runs 2 and 4), static stability $\partial \bar{\Theta} / \partial z$ (runs 1 and 5 ), relative humidity $r_{w}\left(r_{i}\right)$ (runs 8 and 9), and mean vertical wind shear $\partial u_{g} / \partial z, \partial v_{g} / \partial z$ (runs 6 and 7). Runs 10 and 11 use the same external parameters as the control run but elucidate the importance of the radiation term. In simulation 10 , the radiation term is neglected in the droplet growth equation while in run 11 radiative heating is not included both in the droplet growth equation and in the thermodynamic equation. Finally, the sensitivity of numerical results on different choices of the initial ice particle size is investigated. In runs 12 and 13 , we set $r_{e}=4.8$ $\mu \mathrm{m}$ and $r_{e}=1.7 \mu \mathrm{m}$, respectively. 
The integrations run over 1200 time steps up to $t=$ $1800 \mathrm{~s}$. Detailed results will be given in the next section.

\section{Results}

\section{a. Control run}

The flow fields provide an important indication of the nature of the resolved-scale eddies. Figures 1 and 5-8 illustrate typical realizations of the secondary flow pattern in vertical plan views. The figures show isopleths of the various fields, which have been averaged along the airplane flight direction (i.e., along the $y$ direction). Except for $\bar{\Theta}_{v}$, horizontal averages have not been removed. Figure 1 shows contours of the ice content in vertical $x-z$ cross sections at times (a) $t=2 \mathrm{~min}$, (b) $t$ $=4 \min$, (c) $t=8 \mathrm{~min}$, (d) $t=14 \min$, (e) $t=22$ min, and (f) $t=30 \mathrm{~min}$. Isolines are drawn for 1,10 , 20,25 , and $30 \mathrm{mg} \mathrm{kg}{ }^{-1}$. Superimposed are velocity vectors of the resolved-scale velocity field (maximum indicated). Figures 5-8 represent vertical cross sections for the variables $N_{\text {Тот }}$ (particle concentration), $r_{\text {eff }}$ (effective radius), $\bar{w}$, and $\bar{\Theta}_{v}$ for the same times as Fig. 1.

At time zero, the model starts from the equilibrium mean fields already described, except for small random disturbances applied to the temperature field within the contrail. The amplitude of the temperature perturbations is found to increase by instability and, consequently, the contrail grows as well in the horizontal as in the vertical direction. After 2 min the two cloud lines interconnect to form a single cloud band. The flow field is composed of two counterrotating vortices originating at the edges of the contrail. These vortices entrain moist environmental air into the contrail, which is subsequently processed into ice within the cloud. After 30 minutes of simulation time the contrail nearly fills the entire horizontal domain. The maxima of the ice content are found in the central parts of the contrail and amount to about $20 \mathrm{mg} \mathrm{kg}{ }^{-1}$. Throughout the contrail broadening process, the flow field exhibits an increasingly complex structure that is composed of several vortices. The maximum amplitude of the vertical velocity perturbation $\bar{w}$ is of the order of $0.1 \mathrm{~m} \mathrm{~s}^{-1}$ and is found at the upper edges of the contrail.

To get an impression of the three-dimensional structure of the evolving contrail, isosurface plots of the ice content (12 mg kg-1 surface) are displayed in Fig. 2 at (a) $t=4 \mathrm{~min}$, (b) $t=14 \mathrm{~min}$, (c) $t=22 \mathrm{~min}$, and (d) $t=30 \mathrm{~min}$. Interestingly, the ice field is almost homogeneous along the $y$ direction at $t=4 \mathrm{~min}$ and $t=$ $14 \mathrm{~min}$. However, at later times it is striking from the three-dimensional fields that no regular two-dimensional motions are generated by the model. The fact is also supported in Fig. 3, where we have plotted contours of the ice content (left panel) and of the vertical velocity (right panel) in horizontal cross sections (at $z=0 \mathrm{~m}$ ) at times (a) $t=4 \mathrm{~min}$, (b) $t=14 \mathrm{~min}$, and (c) $t=30$ $\min$. At $t=30 \mathrm{~min}$, the ice field as well as the vertical velocity field exhibit considerable variations along the $y$ axis is on scales of about $100 \mathrm{~m}$. Therefore, the assumption of homogeneity along the streamwise direction of two-dimensional studies appears at least questionable on the basis of our three-dimensional results, especially in the mature state of the dispersion regime. The applicability of the two-dimensional approximation is also highlighted in Fig. 4, which shows time series of resolved-scale variances for a three- and a two-dimensional simulation of the control case. Depicted are mean integral variances (per meter along the $y$ direction) of the along-wind $\rho_{0}\left\langle\bar{v}^{\prime \prime 2}\right\rangle$, cross-wind $\rho_{0}\left\langle\bar{u}^{\prime \prime 2}\right\rangle$, and vertical velocity component $\rho_{0}\left\langle\bar{w}^{\prime \prime 2}\right\rangle$. It is evident that the two-dimensional simulation successfully predicts the evolution of the $\bar{u}^{\prime}$ and $\bar{w}^{\prime}$ variance in the initial and the mature phase of the dispersion regime. Both threedimensional and two-dimensional simulations produce a variance ratio $\left\langle\bar{u}^{\prime \prime 2}\right\rangle /\left\langle\bar{w}^{\prime \prime 2}\right\rangle$ of about 2 for $t>1200 \mathrm{~s}$, which is a characteristic feature of semistable-layer turbulence. In contrast, the evolution of the along-wind variance $\left\langle\bar{v}^{\prime \prime}\right\rangle$ is not properly reproduced by the twodimensional model. It appears that for $t>1200 \mathrm{~s}$ threedimensional effects become quite important. At $t=$ $1800 \mathrm{~s}$, the three-dimensional model predicts a resolvedscale variance ratio $\left\langle\bar{u}^{\prime \prime 2}\right\rangle /\left\langle\bar{v}^{\prime \prime 2}\right\rangle$ of about 6 , while the two-dimensional model predicts a ratio of $\left\langle\bar{u}^{\prime \prime 2}\right\rangle$ to $\left\langle\bar{v}^{\prime \prime 2}\right\rangle$ of almost infinity (the ratio $\left(\left\langle\bar{u}^{\prime \prime 2}\right\rangle /\left\langle\bar{v}^{\prime \prime 2}\right\rangle\right)_{2 \mathrm{D}}$ is not identical to infinity since Coriolis forces have been included in the two-dimensional simulation). This supports the suggestion that three-dimensional effects reduce the resolved-scale variance ratio $\left\langle\bar{u}^{\prime \prime}\right\rangle /\left\langle\overline{\boldsymbol{v}}^{\prime \prime}\right\rangle$ because, as a result of the pressure-velocity-gradient correlation $\left\langle\bar{p}^{\prime \prime} \partial \bar{v}^{\prime \prime}\right|$ $\partial y\rangle$, coupling of $\bar{v}^{\prime \prime}$ momentum to $\bar{u}^{\prime \prime}$ and $\bar{w}^{\prime \prime}$ momentum is stronger than two-dimensional models suggest. Therefore, we conclude that even two-dimensional-like phenomena should be modeled with a three-dimensional model to get a proper turbulent kinetic energy evolution. Nevertheless, it turns out that the two-dimensional modeling approach has its justification as the two-dimensional model predicts many aspects of the contrail evolution quite successfully (e.g, vertical velocity variance, ice production rate, and diffusion parameters of the contrail derived from the two-dimensional solution resemble quite closely those of the three-dimensional runs).

Figures 5 and 6 show the ice particle concentration and the effective radius fields from the control run. These microphysical parameters are inferred from the calculated one-dimensional number density function $f(r, \mathbf{x}, t)$ and are obtained with the following relations:

$$
\begin{gathered}
N(\mathbf{x}, t)=\int_{r_{1}}^{r_{2}} f(r, \mathbf{x}, t) d r \\
r_{\text {eff }}(\mathbf{x}, t)=\frac{\int_{r_{1}}^{r_{2}} r^{3} f(r, \mathbf{x}, t) d r}{\int_{r_{1}}^{r_{2}} r^{2} f(r, \mathbf{x}, t) d r},
\end{gathered}
$$



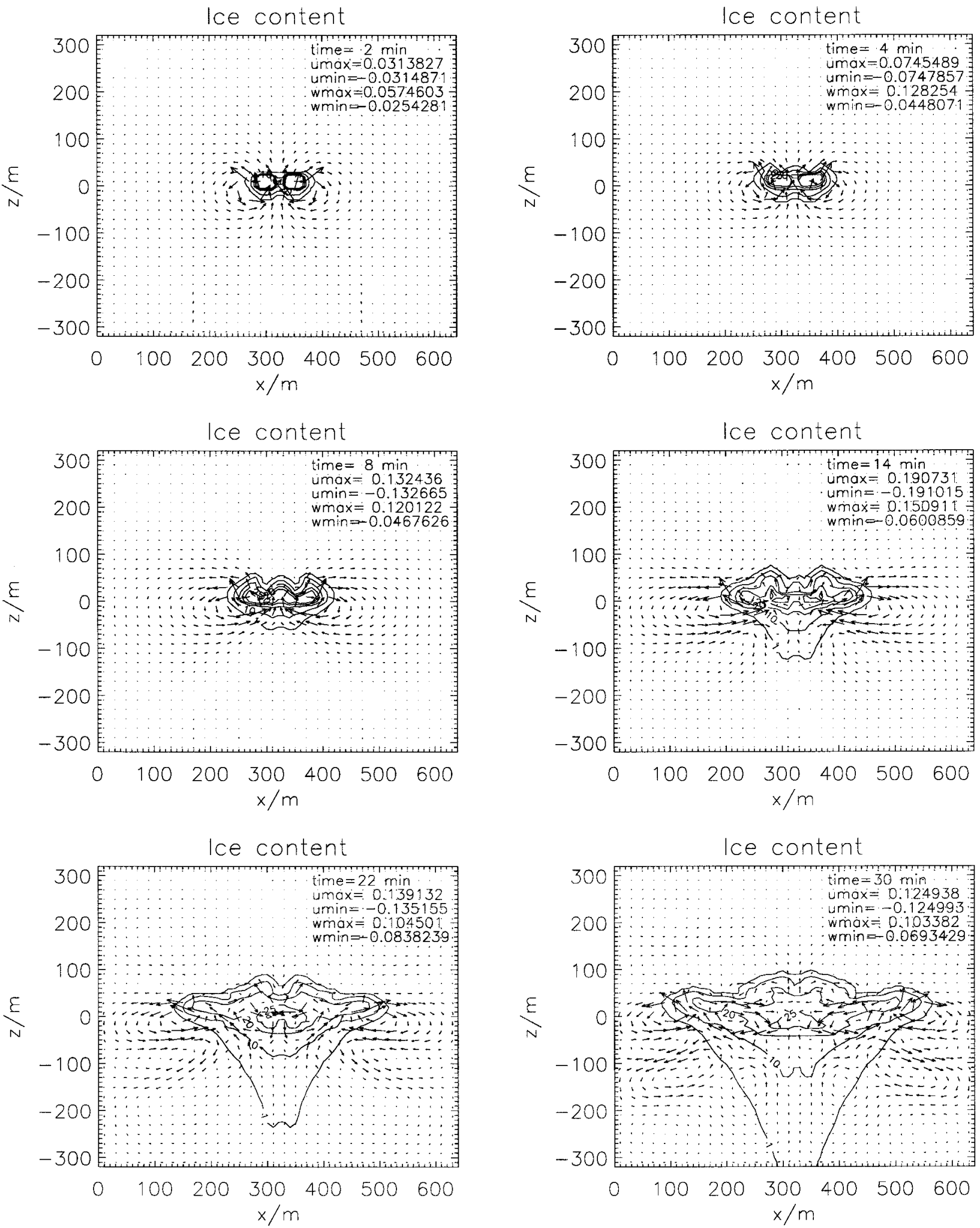

FIG. 1. Contour plots of the along-wind averaged (i.e., along the $y$ direction) ice content in vertical $x-z$ cross sections at times (a) $t=2$ min, (b) $t=4 \min$, (c) $t=8 \mathrm{~min}$, (d) $t=14 \mathrm{~min}$, (e) $t=22 \mathrm{~min}$, and (f) $t=30 \mathrm{~min}$ for the control run. Isolines are drawn for 1,10 , 20,25 , and $30 \mathrm{mg} \mathrm{kg}^{-1}$. Superimposed are velocity vectors of the resolved-scale velocity field (maximum values indicated). 


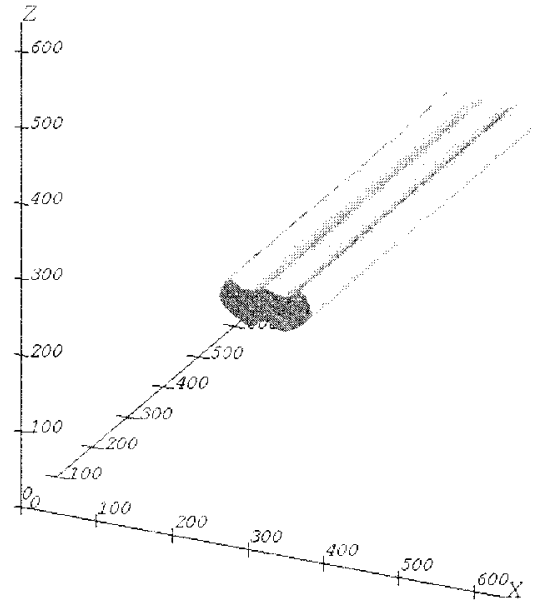

(a)

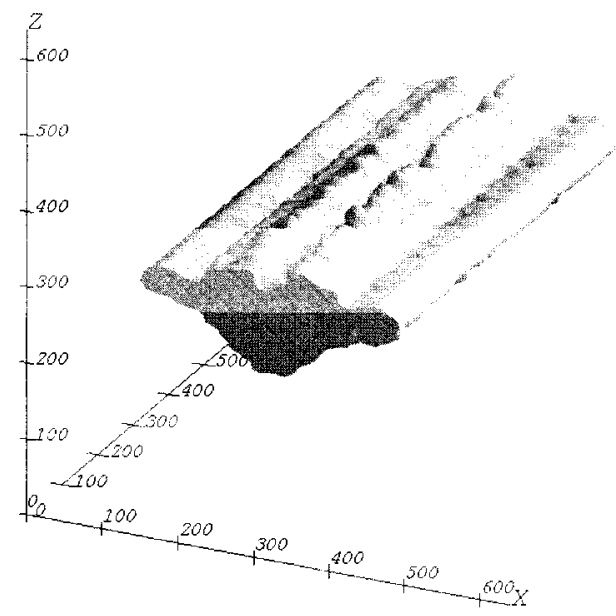

(c)

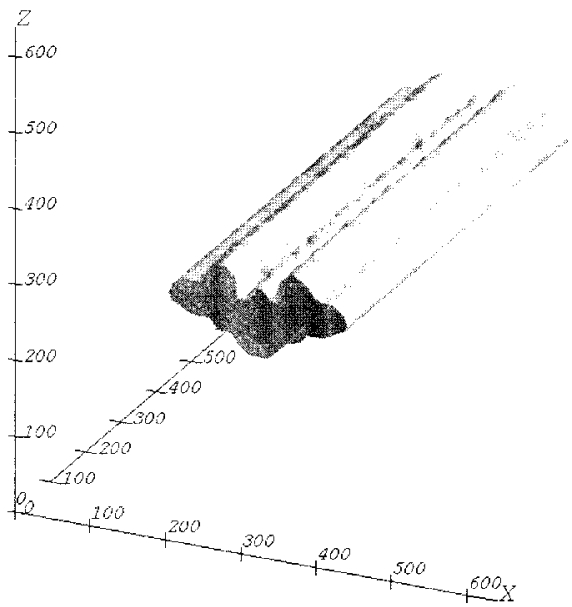

(b)

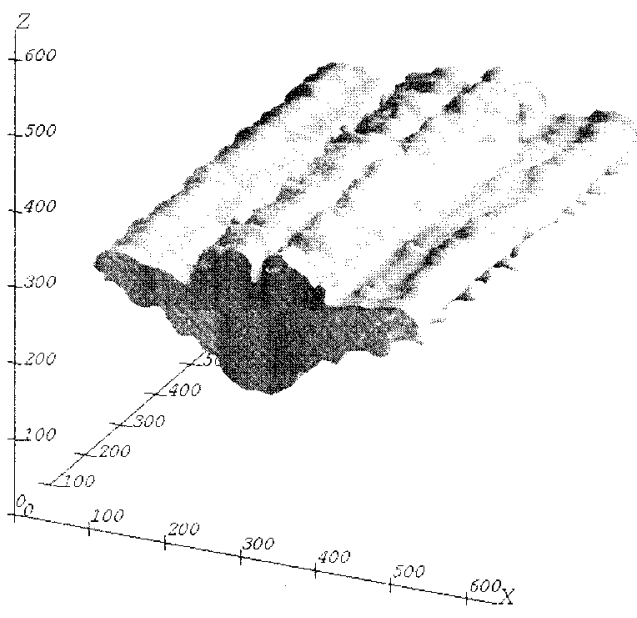

(d)

FIG. 2. Three-dimensional structure of the evolving contrail in the control run. Drawn are isosurface plots of the ice content (12 mg kg $\mathrm{m}^{-1}$ surface) at (a) $t=4 \mathrm{~min}$, (b) $t=14 \mathrm{~min}$, (c) $t=22 \mathrm{~min}$, and (d) $t=30 \mathrm{~min}$. The grid boxes depict the $64 \times 64 \times 64$ computational domain (in a $640 \mathrm{~m} \times 640 \mathrm{~m} \times 640 \mathrm{~m}$ volumetric space).

in which $N(\mathbf{x}, t)$ is the particle concentration and $r_{\text {eff }}$ is the effective radius, respectively, and $r_{1}$ and $r_{2}$ are the upper and lower boundaries of the radius interval over which the particles are calculated $(1 \mu \mathrm{m} \leq r \leq 64 \mu \mathrm{m})$. From the calculations we find that in the central part of the contrail the total concentration of ice particles is largest $\left(N \approx 10^{8} \mathrm{~kg}^{-1}\right)$. Hence, at $z=0 \mathrm{~m}$, which corresponds to the formation level, the ice particle concentration has its maximum and decreases rapidly below $z=-150 \mathrm{~m}$ and above $z=150 \mathrm{~m}$, as shown in Fig. 5. The effective radius field exhibits a clear stratification, as shown in Fig. 6. The smallest ice particles are found in the central region of the contrail, corresponding to the same area where the largest ice content and the maximum ice particle concentration appear. In this zone the particles grow from about $r_{\text {eff }}=2.5 \mu \mathrm{m}$ at $t=0$ min to about $r_{\text {eff }}=6.5 \mu \mathrm{m}$ at $t=30 \mathrm{~min}$. At lower levels, the mean value of the effective radius increases up to about $r_{\text {eff }}=30 \mu \mathrm{m}$. This fact could be explained as follows.

The small ice particles in the central part of the contrail are formed in the early aircraft exhaust plume by homogeneous or heterogeneous nucleation. As the contrail grows by entraining supersaturated air these particles spread over a large area and grow due to vapor deposition. As a result, in the central part of the contrail 

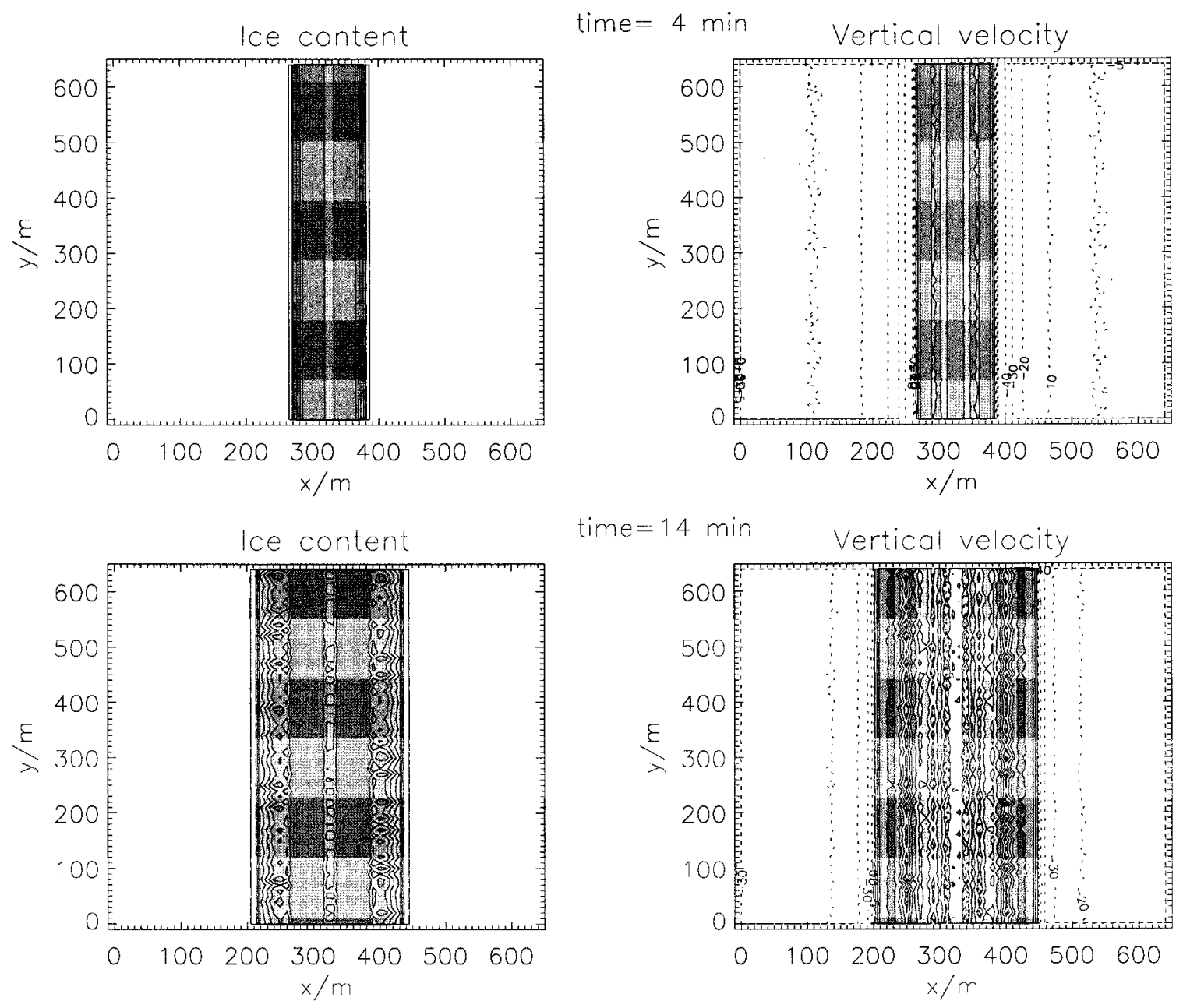

time $=14 \mathrm{~min} \quad$ Vertical velocity
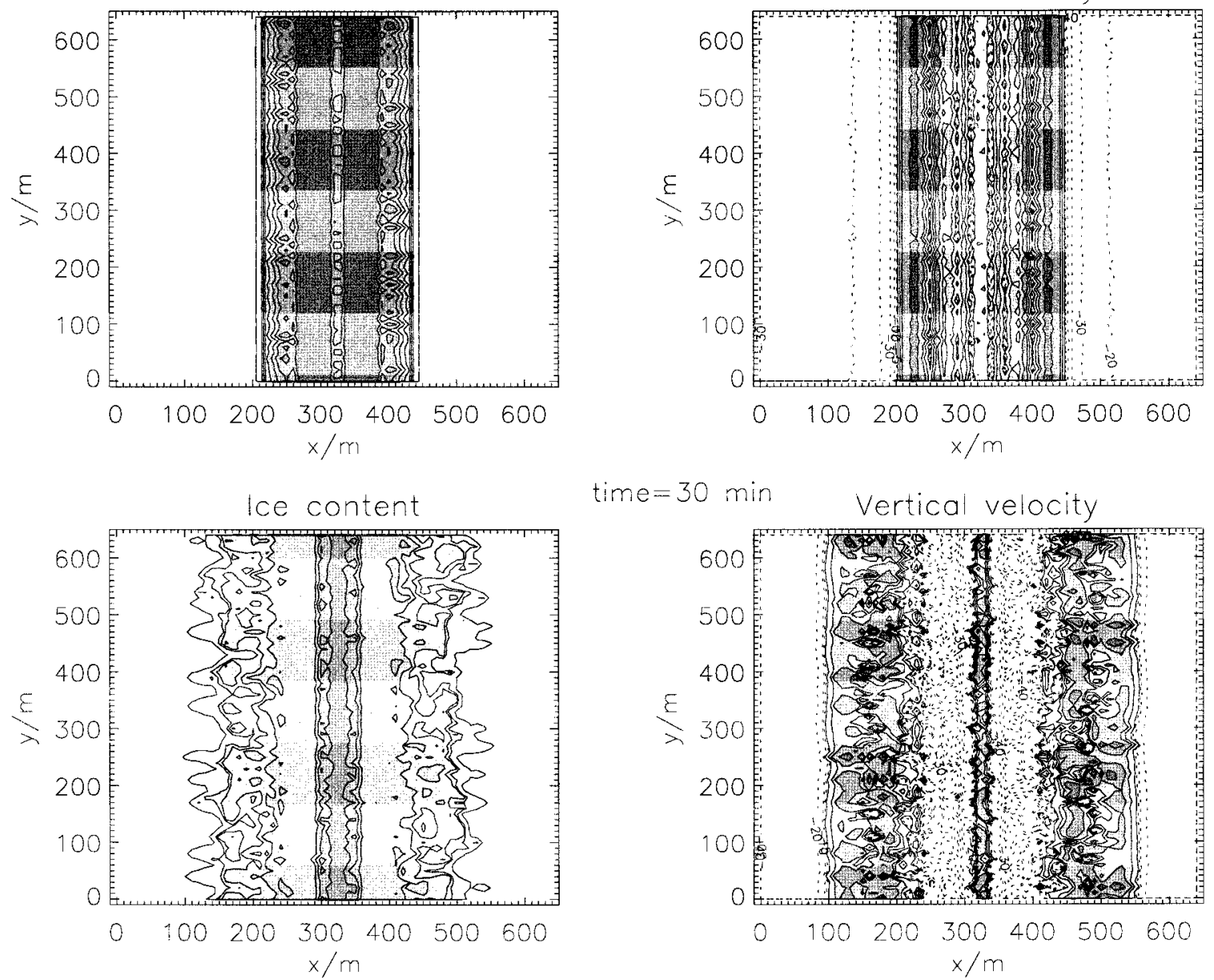

time $=30 \mathrm{~min} \quad$ Vertical velocity

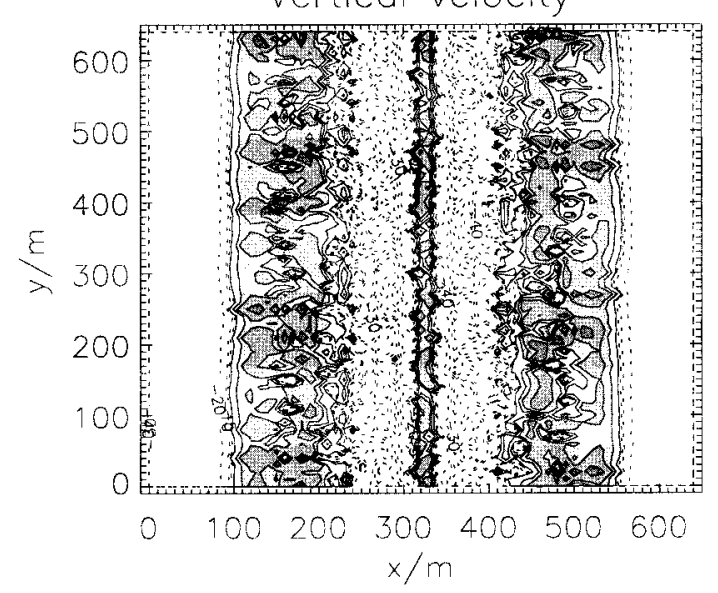

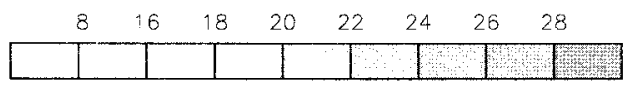

$m g \mathrm{~kg}^{-1}$

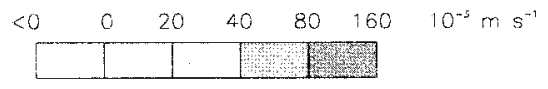

FIG. 3. Contour plots of the ice content (left panel) and of the vertical velocity (right panel) in horizontal $x-y$ cross sections located at $z$ $=0 \mathrm{~m}$ at times (a) $t=4 \mathrm{~min}$, (b) $t=14 \mathrm{~min}$, and (c) $t=30 \mathrm{~min}$ for the control run. Contours for the ice content are drawn for 8,16 , $18,20,22,24,26$, and $28 \mathrm{mg} \mathrm{kg}^{-1}$. Vertical velocity is scaled by using a factor of $10^{3}$. Solid and dashed lines represent, respectively, positive and negative perturbations; isolines correspond to $-40,-30,-20,-10,0,20,40,80$, and $160 \mathrm{~m} \mathrm{~s}^{-1}$. 


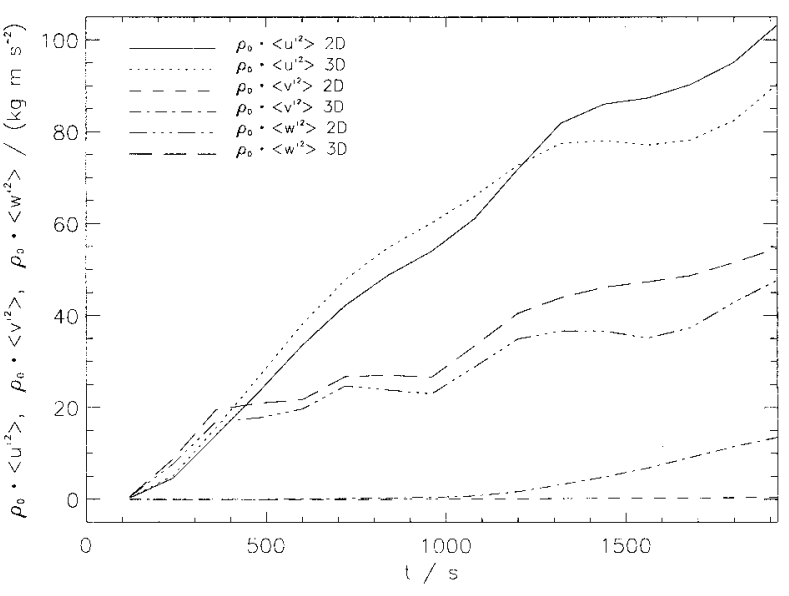

FIG. 4. Time series of resolved-scale variances for a three- and a two-dimensional simulation of the control case. Depicted mean integrated variances (per meter along the $y$ axis) of the along-wind $\rho_{0}$ $\left\langle\bar{v}^{\prime \prime 2}\right\rangle$, cross-wind $\rho_{0}\left\langle\bar{u}^{\prime \prime 2}\right\rangle$, and vertical velocity component $\rho_{0}\left\langle\bar{w}^{\prime \prime 2}\right\rangle$. Definition of line patterns is given in the legend of this figure.

the particle size increases with time and the total particle concentration decreases with time due to dilution. A small fraction of the particles attains a size at which gravitational settling becomes important. Since the largest particles have the largest terminal velocity, the proportion of bigger particles is larger in the lower part of the contrail than in the upper part of the ice cloud. Obviously, the small particles contribute more to the ice content than to the sedimentation rate, resulting in a decrease of the ice content and the total ice particle concentration at lower levels.

To illustrate the dynamical and thermodynamical features of the contrail evolution we show vertical cross sections of the vertical velocity and virtual potential temperature anomaly fields at different times (Figs. 7 and 8). During the first stage of contrail development, which occurs during the first 8 min of simulation time, the updraft velocity pattern shows a w-shaped pattern within the contrail, as a result of the developing counterrotating vortices. These vortices produce updrafts with maxima of about $0.1 \mathrm{~m} \mathrm{~s}^{-1}$ within the contrail. These maxima are due to the local extrema of the horizontal gradient of buoyancy caused by the vapor deposition process creating a source of horizontal vorticity. The second stage of contrail evolution comprises the time interval from 14-30 min. During this period the vertical velocity field shows different distinct maxima within the cloud. These maxima are located near the outer edge of the ice cloud with values of about $0.1 \mathrm{~m}$ $\mathrm{s}^{-1}$. These maxima are coupled with downdraft velocities near the same levels inside and outside the cloud. This velocity configuration is due to the developing complex multiple vortex structure as a result of the continual production of vorticity at the cloud boundary.

The fields of virtual potential temperature anomaly shown in Fig. 8 are seen to be well correlated with the field of vertical velocity. At every time the field of ver- tical velocity is, for the most part, in phase with the temperature perturbation field, that is, maximum temperature perturbations coincide with strongest upward motions and vice versa, indicating an upward buoyancy flux. Maximum temperature perturbations are found to be in the range between 0.04 and $0.06 \mathrm{~K}$ and are determined largely by the vapor deposition process due to latent heat release. Thus, we anticipate that the turbulent kinetic energy of the contrail is produced by buoyancy, which is reinforced by condensational heating. In principle, differential radiative cooling could also provide a substantial source, via buoyancy production, for turbulent kinetic energy of the contrail. However, our numerical simulations of contrail development have revealed a surprising insensitivity to longwave radiative cooling/heating (see also next subsection). This is due to the small cooling rates that we found in the contrail compared to the much higher values present, for instance, in stratocumulus clouds. Inspection of the longwave radiation cooling field reveals (not shown here) that the cooling is almost homogeneously distributed within the contrail with maximum cooling rates of about $-1 \mathrm{~K} /$ day (by comparison: maximum infrared cooling rates at cloud top of stratocumulus clouds are about $-100 \mathrm{~K} /$ day). Therefore, we conclude that in contrast to the stratocumulus-topped planetary boundary layer, which is mainly driven by cloud-top radiation cooling, cloud-radiation interaction insignificantly influence the dynamics of contrails and that latent heating due to vapor deposition provides the important energy source for turbulent kinetic energy of contrails. However, despite our findings about the insensitivity of contrail dynamics on radiation, one should keep in mind that this statement is only valid on a timescale of about $30 \mathrm{~min}$. On a longer timescale, on the other hand, one should observe thin cirrus/contrail lifting/sinking even due to small heating/ cooling effect of importance for contrail evolution in its very mature stage (Jensen et al. 1996). Moreover, there is no doubt that thin cirrus clouds can be a significant factor in affecting the radiative energy balance of the earth and in turn may have the potential to lead to greenhouse warming (e.g., Prabhakara et al. 1993).

\section{b. Sensitivity runs}

In order to examine the influence of external parameters, initial conditions, and radiation on the development of contrails, sensitivity tests have been performed. The control run was rerun (see also Table 1) with

1) different ambient temperatures $T_{00}$ (runs 2 and 4);

2) different static stabilities $\partial \bar{\Theta} / \partial z$ (runs 1 and 5);

3) different relative humidity $r_{w}\left(r_{i}\right)$ (runs 8 and 9);

4) mean vertical wind shear (runs 6 and 7);

5) no radiation term in the droplet growth equation and no longwave radiative effects at all (runs 10 and 11); and

6) different initial ice particle sizes (runs 12 and 13). 

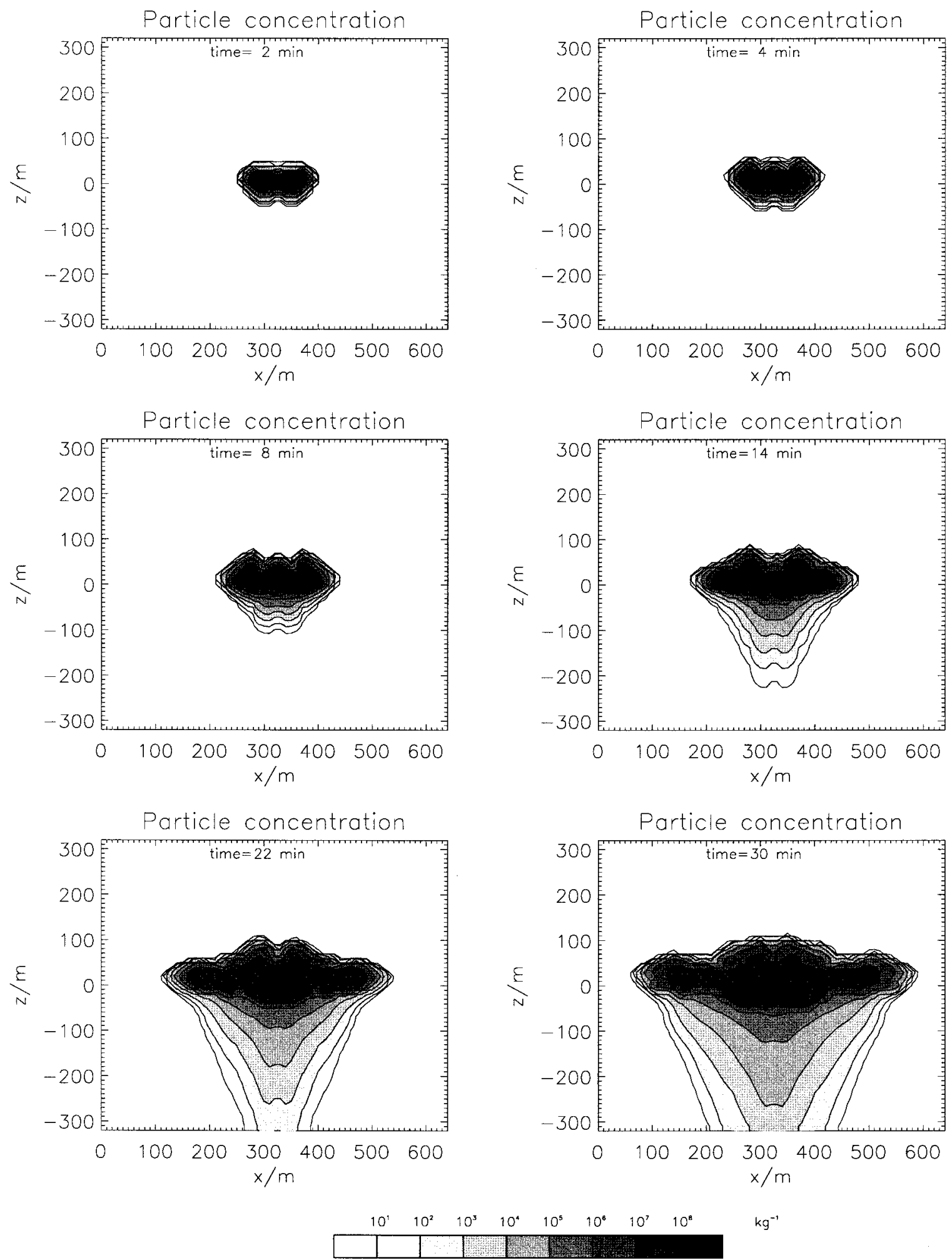

FIG. 5. Contour plots of the ice particle concentration field. Isolines are drawn for $10^{1}, 10^{2}, 10^{3}, 10^{4}, 10^{5}, 10^{6}, 10^{7}$, and $_{10}^{8} \mathrm{~kg}^{-1}$. Other features as in Fig. 1. 

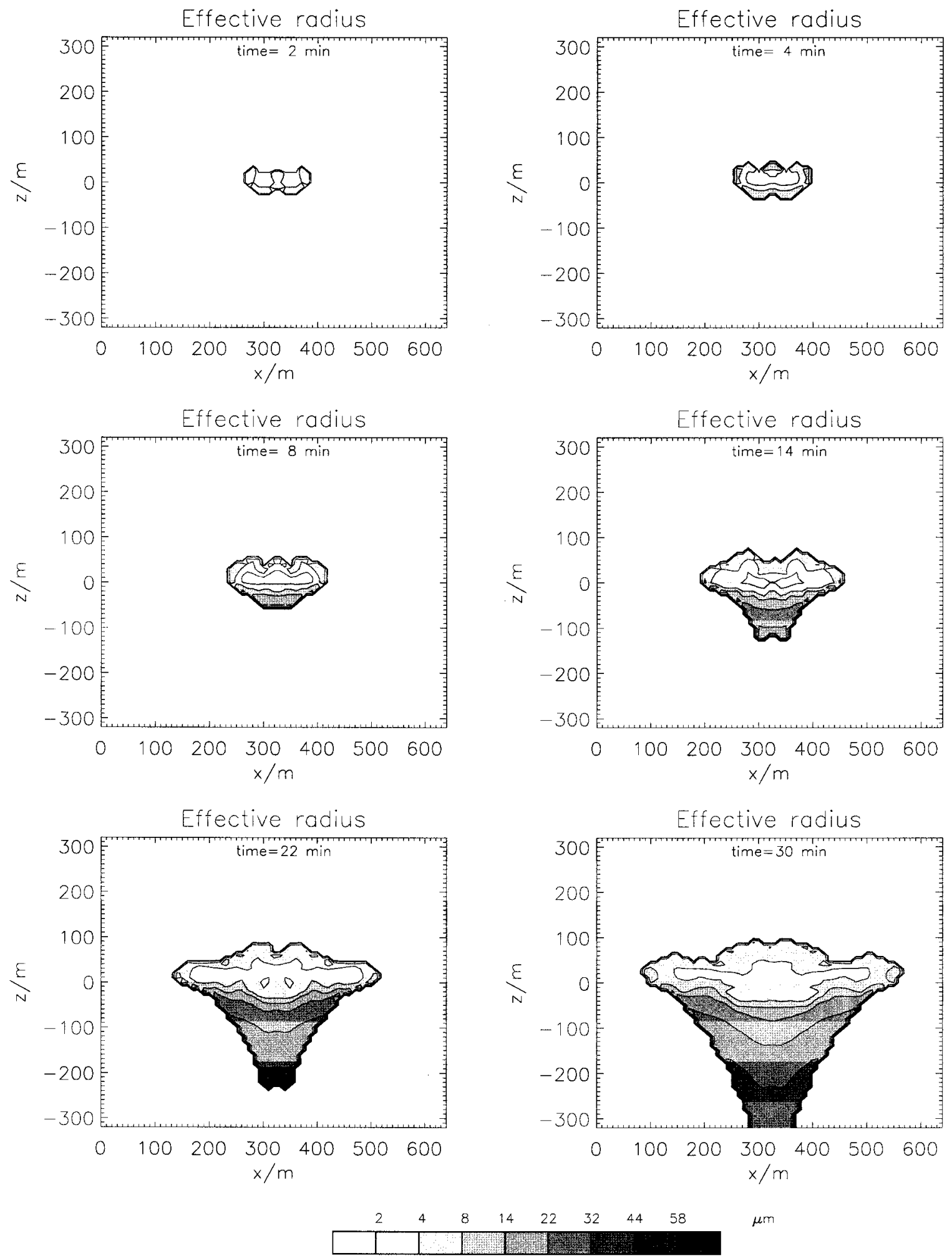

Fig. 6. Contour plots of the effective radius field. Isolines are drawn for 2, 4, 8, 14, 22, 32, 44, and $58 \mu \mathrm{m}$. Other features as in Fig. 1. 

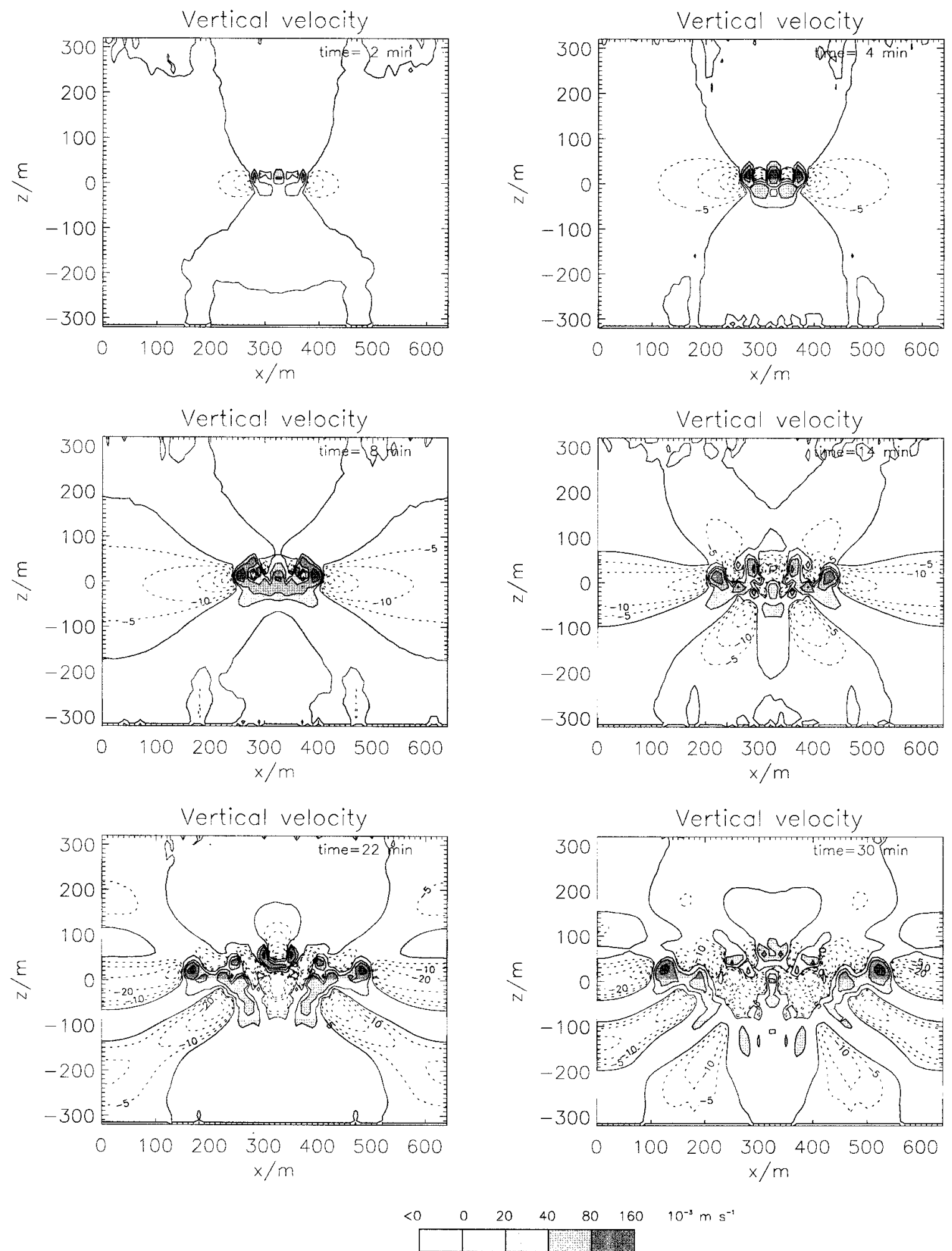

FIG. 7. Contour plots of the vertical velocity field. Vertical velocity is scaled by using a factor of $10^{3}$. Solid and dashed lines represent, respectively, positive and negative perturbations; isolines correspond to $-40,-30,-20,-10,-5,0,10,20,40,80$, and $160 \mathrm{~m} \mathrm{~s}^{-1}$. Other features as in Fig. 1. 

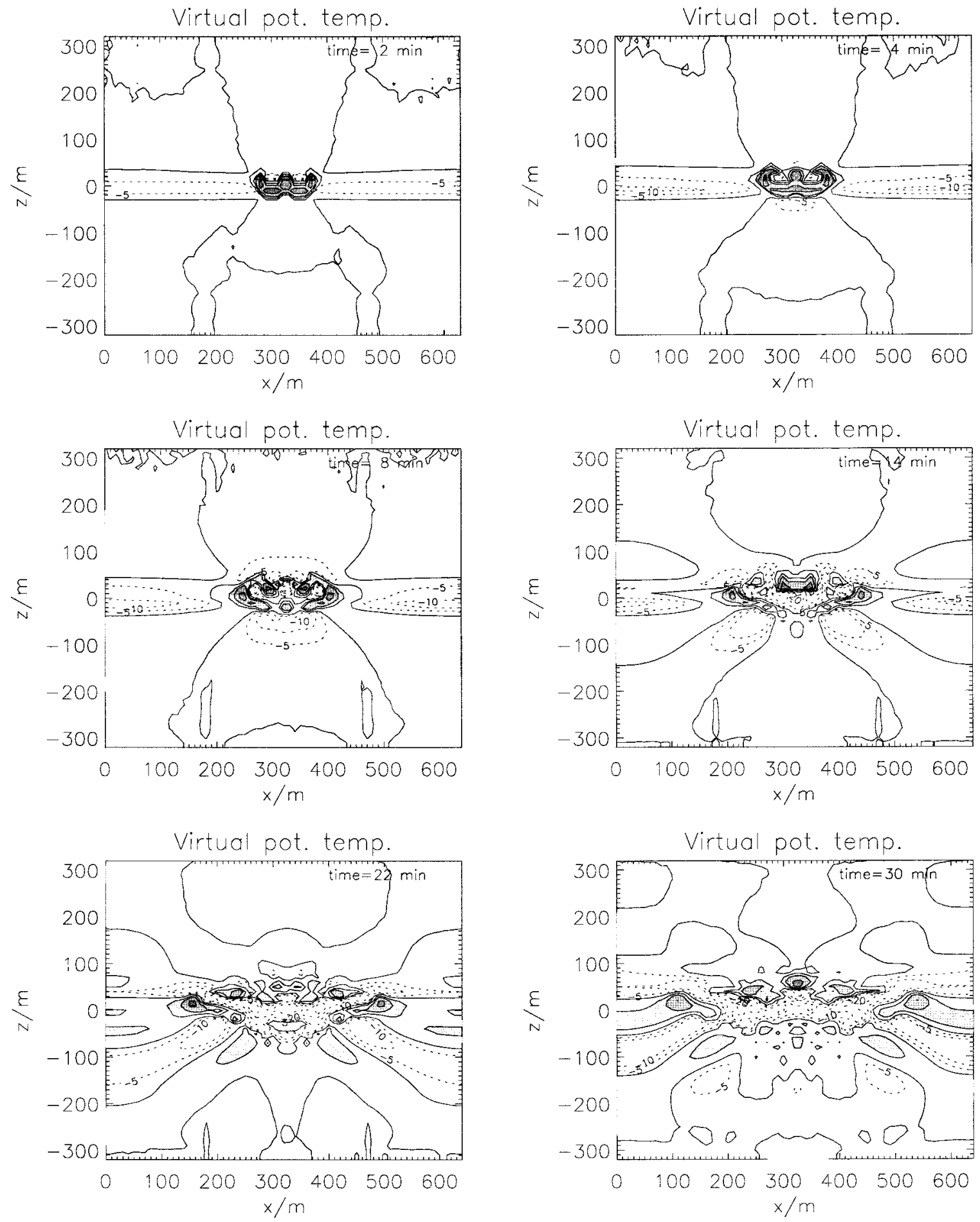

$\begin{array}{llllllll}<0 & 0 & 10 & 20 & 40 & 80 & 160 & 10^{-3} \mathrm{~K}\end{array}$

FIG. 8. Contour plots of the virtual potential temperature anomalies. Virtual potential temperature is scaled by using a factor of $10^{3}$. Solid and dashed lines represent, respectively, positive and negative perturbations; isolines correspond to $-40,-30,-20,-10,-5,0,10,20$, 40, 80, and $160 \mathrm{~K}$. Other features as in Fig. 1. 
In Fig. 9 we have plotted integral quantities such as domain-averaged turbulent kinetic energy (a), integrated ice content per meter of flight (b), horizontal spreading $\sigma_{x}^{2}(\mathrm{c})$, and vertical spreading $\sigma_{z}^{2}$ (d) of the contrail as function of time for the various model experiments. These quantities are all inferred from the simulations and are obtained with the following relations

$$
\mathrm{KE}=\frac{\frac{1}{2} \iiint_{v}\left(\bar{u}^{\prime \prime 2}+\bar{v}^{\prime \prime 2}+\bar{w}^{\prime \prime 2}\right) d x d y d z}{\iiint_{v} d x d y d z}
$$

where $\bar{u}^{\prime \prime}, \bar{v}^{\prime \prime}$, and $\bar{w}^{\prime \prime}$ denote the deviations of the velocity components from their horizontal means and the integration is performed over the total model domain. In order to quantify the horizontal and vertical spreading rates of a contrail it is necessary to define effective contrail cross sections $\bar{A}$ by

$$
\bar{A}(t)=\frac{1}{L_{y}} \int_{0}^{L_{y}}\left(\iint_{C(y, t)} d x d z\right) d y,
$$

where $C(y, t)$ denotes the area enclosed by the contour $N_{\text {min }}^{*}=100 \mathrm{~kg}^{-1}$ of those particles that are insignificantly influenced by gravitational settling, that is,

$$
C(y, t)=\left\{\begin{array}{c}
r_{o}=8 \mu \mathrm{m} \\
\left.(x, z) ; \quad \int f(r, \mathbf{x}, t) d r \geq N_{\min }^{*}\right\} . \\
r_{u}=1 \mu \mathrm{m} \\
\sigma_{x}(t)=\frac{1}{2} P_{x}(\bar{A}(t)) \\
\sigma_{z}(t)=\frac{1}{2} P_{z}(\bar{A}(t)) .
\end{array}\right.
$$

The horizontal and vertical half-widths of the contrail are then obtained by taking the half of the projections of the effective contrail cross section on the $x$ and $z$ axis, respectively, that is,

$$
\begin{aligned}
& \sigma_{x}(t)=\frac{1}{2} P_{x}(\bar{A}(t)) \\
& \sigma_{z}(t)=\frac{1}{2} P_{z}(\bar{A}(t)) .
\end{aligned}
$$

As seen in Fig. 9, the evolution of contrails is mainly controlled by relative humidity, temperature, and static stability of the ambient air. The greatest reductions in turbulent kinetic energy occur in runs 2 and 8, where the TKE has dropped from $0.8 \times 10^{-3}$ to $0.17 \times 10^{-3}$ and $0.8 \times 10^{-4} \mathrm{~m}^{2} \mathrm{~s}^{-2}$. The physical reason for this effect arises from the microphysical processes govern- ing the diffusional growth of ice particles. Inspection of Eq. (24) reveals that the mass growth rate of a single ice particle is directly proportional to the ambient relative humidity and that for a given relative humidity the growth rate is a monotonic increasing (stronger than linear) function of temperature. As a result, reducing the relative humidity of the ambient air by $12 \%$ (run 8 ) or lowering the temperature of the environmental air by $10 \mathrm{~K}$ (run 2) produces less vigorous eddies. In both cases the conversion rate from water vapor to ice, and hence the latent heat release, is much less than in the reference run owing to the initial decrease of total water content. On the other hand, increasing the environmental humidity by $12 \%$ (run 9) or raising the environmental temperature by $10 \mathrm{~K}$ (run 4) results in enhanced energy levels. In both cases the conditionally available potential energy that could by converted into kinetic energy is larger than in the reference case. Therefore, we conclude that vapor deposition provides the significant energy source driving the secondary flow eddies within contrails. The influence of thermal stratification is also very pronounced. In the simulation that has been performed in a neutral stratified atmosphere (run 1) enhanced energy levels and greater ice production rates are found. The physical reason for this effect is due the fact that in this case the eddies have to perform no work against the stable stratification. On the other hand, for a more stably stratified (relative to the reference run) environment (run 5), the stratification counteracts vertical motions so that mixing is much slower in the vertical than in the horizontal directions. The ratio of horizontal to vertical variance as a measure of anisotropy amounts to about $2: 1$ in the control case and about $4: 1$ in run 5 . These values for the anisotropy ratio are typical for turbulence in a stably stratified medium where rather large and almost horizontally oriented eddies cause the mixing (Gerz and Schumann 1991; Schumann and Gerz 1995). Recently, Schumann et al. (1995) analyzed atmospheric turbulence data from a measurement campaign in the North Atlantic flight corridor near the tropopause. They also found strong anisotropic air motions with almost vanishing turbulent dissipation rates, suggesting that the spectral turbulent energy transfer from larger to smaller scales was almost suppressed, or even may go upscale to form larger eddies from smaller eddies, as hypothesized for quasi-two-dimensional stable layer turbulence (Gage 1979; Lilly 1983, 1988). Figure 9 depicts the ice production rate as only slightly dependent on the initial ice particle concentration. The comparison reveals only a small change of the integrated ice content IC at $t=1800 \mathrm{~s}\left(3.6 \times 10^{-1} \mathrm{~kg} \mathrm{~m}^{-1}\right.$ (run 12 ), and $4.7 \times 10^{-1} \mathrm{~kg} \mathrm{~m}^{-1}$ (run 13 ) versus $4.4 \times 10^{-1}$ $\mathrm{kg} \mathrm{m}^{-1}$ in the reference run), although the initial ice particle concentration has been varied by a factor of $1 / 8$ (run 12) and 2.8 (run 13), respectively. The insensitivity may be explained by an overseeding of numerous ice crystals that will grow more slowly than a single ice particle owing to the competition of many ice crystals 
(a) TKE
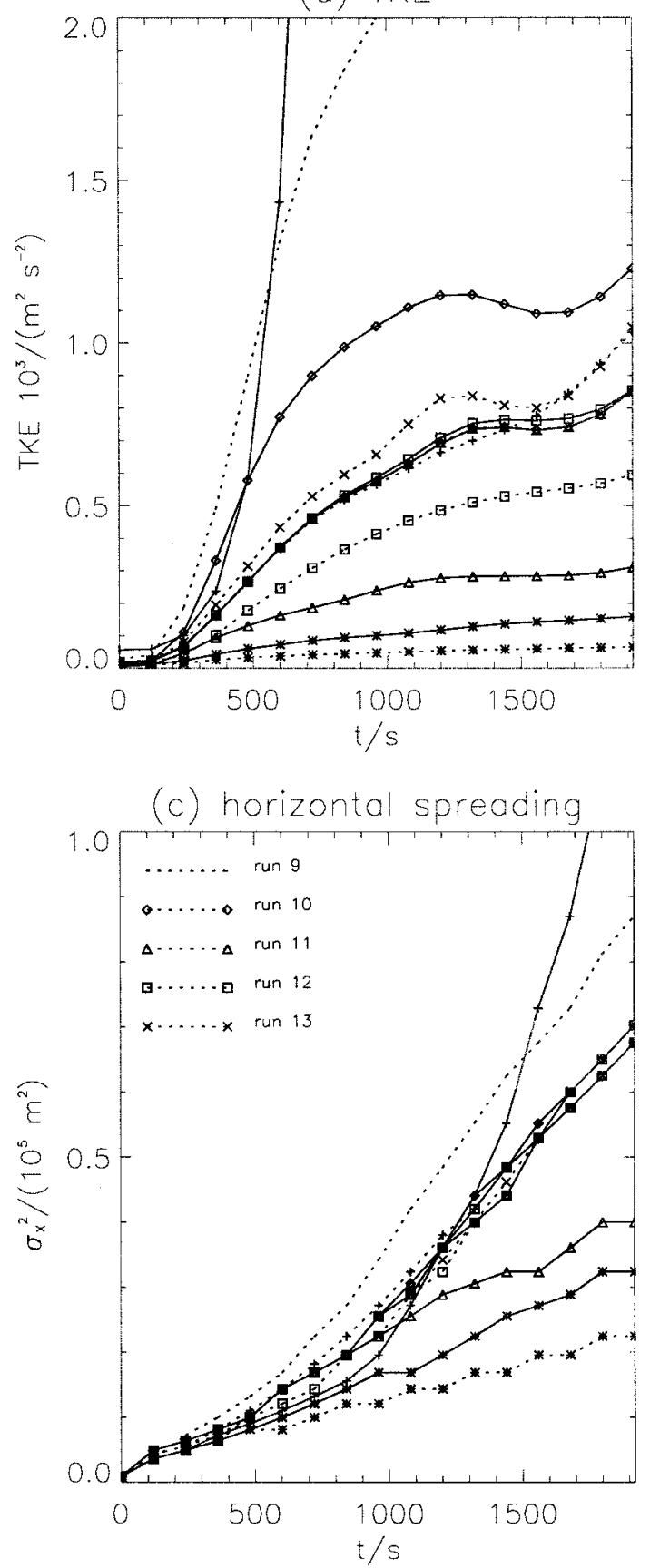

(b) 10
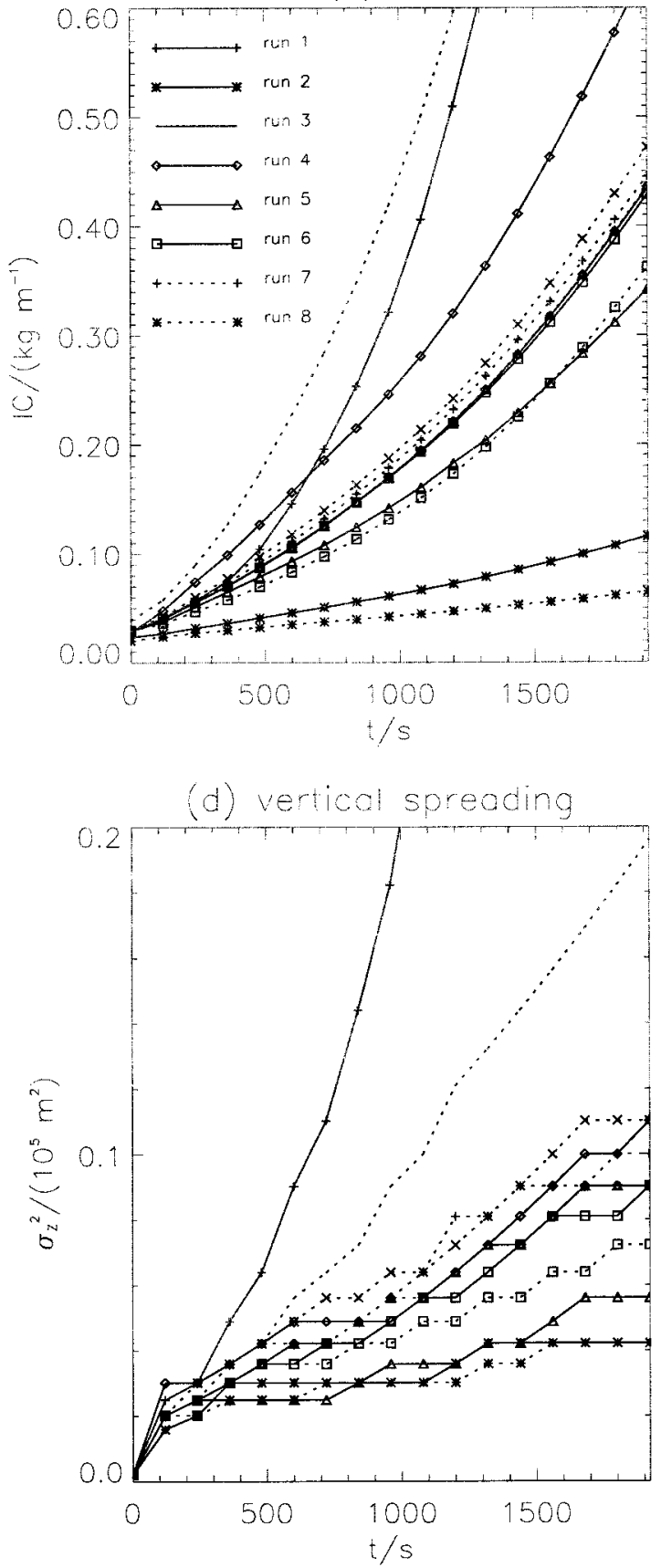

FIG. 9. Evolution of domain-averaged turbulent kinetic energy, TKE(a), total ice content, IC (b), square of the horizontal half width, $\sigma_{x}^{2}$ (c), and square of the vertical half width, $\sigma_{z}^{2}(\mathrm{~d})$, for the various model runs. Definition of symbols are given in the legend of (b) and (c).

for the available water vapor. The results for the runs $6,7,10$, and 11 are nearly indistinguishable from the control run, that is, the mean vertical wind shear of the horizontal velocity components and radiative processes are of minor importance in the evolution of contrails at least during the 30-min simulation period. With respect to runs 6 and 7 we have to admit that these experiments may have only restricted applicability to the question of the potential importance of vertical shear since the assumed shear cases contained only very weak wind shear $\left[5 \times 10^{-3} \mathrm{~s}^{-1}\right.$ (along-wind shear); $1 \times 10^{-3} \mathrm{~s}^{-1}$ (cross-wind shear)]. Estimates of diffusion parameters of aircraft exhaust plumes near the tropopause from nitric oxide and turbulence measurements indicate that shear dominates the dispersion after a time of about 1 hour, even for the cases with only a weak wind shear 
TABLE 2. Values of horizontal and vertical diffusion coefficients for the various model runs.

\begin{tabular}{|c|c|c|c|c|c|c|c|c|c|c|c|c|c|}
\hline & \multicolumn{13}{|c|}{ Run } \\
\hline & 1 & 2 & 3 & 4 & 5 & 6 & 7 & 8 & 9 & 10 & 11 & 12 & 13 \\
\hline$D_{h}\left(\mathrm{~m}^{2} \mathrm{~s}^{-1}\right)$ & $9.6-48.2$ & 9.0 & 23.9 & 23.9 & 10.5 & 23.9 & 23.9 & 5.6 & 27.6 & 23.9 & 23.9 & 23.9 & 23.9 \\
\hline$D_{v}\left(\mathrm{~m}^{2} \mathrm{~s}^{-1}\right)$ & $9.6-48.2$ & 0.56 & 2.0 & 2.7 & 1.0 & 2.0 & 2.0 & 0.37 & 5.3 & 2.0 & 2.0 & 1.7 & 2.7 \\
\hline
\end{tabular}

of $2 \times 10^{-3} \mathrm{~s}^{-1}$ (Schumann et al. 1995). The effect of radiative transfer on the particle condensational growth is almost negligible since the ice particles are too small on the average. Effects of radiation on ice crystal growth are expected to be important only for particles having sizes of more than a few tens of microns (Gierens 1994). Moreover, radiative cooling leads to insignificant changes in the structure of the modeled contrail. This is due to (as already discussed in the previous subsection) the small cooling rates that we found in contrails. The impact of radiative processes on deposition growth and thermodynamic properties may be important when cloud radiative cooling is significant as in boundary layer clouds (Ackerman et al. 1995).

Figures 9c and 9d depict the development of the contrail size in terms of squares of its horizontal and vertical half widths. In comparing the evolution of $\sigma_{x}^{2}$ and $\sigma_{z}^{2}$ we find that the horizontal spreading is, for all cases except run 1, much larger than in the vertical direction. The difference amounts to about one order of magnitude. Moreover, it is notable that the contrail grows vertically quite slowly after a relatively strong expansion initially. The squares of the horizontal and vertical half widths are (except for run 1) almost linear functions of time for $t \geq 500 \mathrm{~s}$. Hence, the spreading of the contrail could be modeled as a turbulent diffusion process according to

$$
\sigma_{x}^{2}=2 D_{h} t+\sigma_{x, 0}^{2}, \quad \sigma_{z}^{2}=2 D_{v} t+\sigma_{z, 0}^{2},
$$

where $t$ is time, $\sigma_{x, 0}$ and $\sigma_{z, 0}$ denote the initial horizontal and vertical half-widths, and $D_{h}$ and $D_{v}$ are the hori-

TABLE 3. Bulk microphysical parameters of simulated contrails after $30 \mathrm{~min}$ of integration time for the various model runs. Listed are mean and maximum values of ice-liquid water content $q_{\mathrm{i}}$ and the ice particle concentration $N_{\text {тот }}$ and the mean effective radius $\left\langle r_{\text {eff }}\right\rangle$.

\begin{tabular}{|c|c|c|c|c|c|}
\hline Run & $\frac{q_{i}}{\left(\mathrm{mg} \mathrm{kg}^{-1}\right)}$ & & $\frac{N_{\mathrm{TOT}}}{\left(\mathrm{kg}^{-1}\right)}$ & & $\frac{\left\langle r_{\text {eff }}\right\rangle}{(\mu \mathrm{m})}$ \\
\hline 1 & $12.5 \ldots 30.3$ & $5.40 \times$ & $10^{6} \ldots 3.8$ & $\times 10^{7}$ & 10.8 \\
\hline 2 & $7.2 \ldots 24.9$ & $2.80 \times$ & $10^{7} \ldots 2.9$ & $\times 10^{8}$ & 5.9 \\
\hline 3 & $10.9 \ldots 28.4$ & $1.43 \times$ & $10^{7} \ldots 2.17$ & $\times 10^{8}$ & 9.8 \\
\hline 4 & $13.6 \ldots 31.0$ & $1.14 \times$ & . . 1.66 & $\times 10^{8}$ & 11.7 \\
\hline 5 & $10.0 \ldots 34.2$ & $1.67 \times$ & $10^{7} \ldots 3.78$ & $\times 10^{8}$ & 10.2 \\
\hline 6 & $10.6 \ldots 28.3$ & $1.41 \times$ & $10^{7} \ldots 2.15$ & $\times 10^{8}$ & 9.8 \\
\hline 7 & $10.2 \ldots 29.7$ & $1.30 \times$ & $10^{7} \ldots 2.38$ & $\times 10^{8}$ & 10.1 \\
\hline 8 & $6.6 \ldots 28.7$ & $3.88 \times$ & $10^{7} \ldots 3.40$ & $\times 10^{8}$ & 4.6 \\
\hline 9 & $18.1 \ldots 36.2$ & $1.08 \times$ & $10^{7} \ldots 1.4$ & $\times 10^{8}$ & 13.0 \\
\hline 10 & $10.9 \ldots 28.4$ & $1.42 \times$ & $10^{7} \ldots 2.17$ & $\times 10^{8}$ & 9.9 \\
\hline 11 & $10.9 \ldots 28.4$ & $1.42 \times$ & $10^{7} \ldots 2.17$ & $\times 10^{8}$ & 9.9 \\
\hline 12 & $9.2 \ldots 29.2$ & $1.80 \times$ & $10^{6} \ldots 2.8$ & $\times 10^{7}$ & 15.4 \\
\hline 13 & $11.5 \ldots 28.9$ & $3.90 \times$ & $10^{7} \ldots 6.3$ & $\times 10^{8}$ & 7.8 \\
\hline
\end{tabular}

zontal and vertical diffusion coefficients, respectively, which are assumed to be constant (Monin and Yaglom 1971). Table 2 shows the values of the diffusion coefficients for all runs obtained by least square fits. For the majority of the runs we find diffusion coefficients of $D_{h}$ $=23.9 \mathrm{~m}^{2} \mathrm{~s}^{-1}$ and $D_{v}=2.0 \mathrm{~m}^{2} \mathrm{~s}^{-1}$. The coefficients depend strongly, as one would expect from the previous discussion on turbulent kinetic energy and total ice content, on humidity, temperature, and static stability of the ambient air. Hence, the computed horizontal diffusion coefficients range between 5.6 and $48 \mathrm{~m}^{2} \mathrm{~s}^{-1}$, depending on the environmental conditions. Likewise the vertical diffusivity coefficients are in the range between 0.37 and $48 \mathrm{~m}^{2} \mathrm{~s}^{-1}$. Recently, Schumann et al. (1995) gave estimates of diffusion parameters of aircraft exhaust plumes based on in situ flight measurements in the North Atlantic flight corridor in the tropopause region. They found strong anisotropic air motions with weak vertical velocity fluctuations. The horizontal diffusivity was estimated as between 5 and $20 \mathrm{~m}^{2} \mathrm{~s}^{-1}$ and the vertical diffusivity exhibits values between 0 and $0.6 \mathrm{~m}^{2} \mathrm{~s}^{-1}$. Our results are in good agreement with these data, provided that the atmospheric situation is characterized by low temperatures (run 2), slight supersaturations (run 8), and moderately stable stratification (run 5), as was likely for the flight route conditions that the observational results are based on.

Except for in situ measurements and remote sensing observations by Knollenberg (1992), Baumann et al. (1993), Schumann (1994), Freudenthaler et al. (1995) and Gayet et al. (1996), little is known about the spatial structure of microphysical and dynamical parameters of contrails. Gayet et al. (1996) analyzed natural and contrail-induced cirrus from in situ and remote sensing measurements during the International Cirrus Experiment (ICE) over the North Sea during fall 1989. These observations were made in a situation of moderate wind shear in a supersaturated (with respect to ice) layer at an altitude of about $8000 \mathrm{~m}$ and at temperatures of about $-37^{\circ} \mathrm{C}$. Although the situation during the observations is comparable to those considered here, none of our results completely fits the situation during the measurements of this case study. Nevertheless, we use these data, and an attempt has been made to perform a rough comparison of our results to these observations. For this purpose we have summarized in Table 3 mean [defined in terms of the area defined in Eq. (37)] and maximum values of bulk parameters characterizing the microphysical properties of the simulated contrails for the various model experiments. 
Simulated mean and maximum ice contents are in excellent agreement with the observations of Gayet et al. (1996), who reported values in the range between 2 and $18 \mathrm{mg} \mathrm{m}^{-3}$ (note that the values in Table 3 have to be multiplied by the density of air $\rho_{00}=0.396 \mathrm{~kg} \mathrm{~m}^{-3}$ to obtain ice contents and ice particle concentrations per volume), whereas the computed values lie between 2.6 and $14.3 \mathrm{mg} \mathrm{m}^{-3}$. In contrast, there seems to be an underprediction of mean effective radii. However, keeping in mind the uncertainties in experimental estimates of microphysical parameters, our results appear also quite realistic with respect to the particle size, that is, values of $\left\langle r_{\text {eff }}\right\rangle$ in the range between 4.6 and $15.4 \mu \mathrm{m}$ in the simulations versus 15 and $18 \mu \mathrm{m}$ in the observations. The most glaring discrepancy between the model and the data is the high particle number density in the simulations. The ICE data suggest ice particle concentrations up to $8.2 \times 10^{5} \mathrm{~m}^{-3}$ within contrails, whereas the computed particle number densities are in the range between $7 \times 10^{5}$ and $2.5 \times 10^{8} \mathrm{~m}^{-3}$. Several possible explanations may be given for the discrepancies between this type of simulation and the observations. These include missing simulated microphysical processes, unawareness about the initial and environmental conditions, and uncertainties about experimental estimates of microphysical parameters.

With respect to the first point a few comments on the limitations of our model are appropriate at this point. A possible limitation of the model results from the assumption of spherical ice particles, and more seriously, from neglect of the aggregation process. As already pointed out in section $2 \mathrm{~b}$, it appears not very likely that aggregation constitutes a significant process in ice clouds at low temperatures. However, more recent results and observations suggest that aggregation may be of more importance than previously thought (Kajikawa and Heymsfield 1989; Mitchell 1988, 1991, 1994). In fact, aggregation could probably establish an important sink for small particles and would increase the number density of large crystals in our model. Hence, we conclude that the potential importance of the aggregation process is an obvious uncertainty in our simulations, but we think that the omission of this process can explain the discrepancy between simulated and measured particle concentrations only in part. However, further observational evidence is required for a truly definite evaluation.

The second possibility is that errors in the assumed initial and environmental conditions significantly altered the simulation. It should be realized that the observations of contrails during ICE possibly cannot be generalized for all cases of this type of clouds, especially for young contrails. In fact, the intercomparison of simulations and observations is hampered by the lack of information, first, about the initial particle size distribution, second, about the amount of water vapor in excess of ice saturation, and, third, about the age and, therefore, about the state of evolution of the observed contrail. Finally, we should note that the apparent discrepancy may be partly traced back to the fact that the measured particle concentrations include contributions from ice crystals with dimensions larger than the PMS probe detection limit $(3 \mu \mathrm{m})$ while the model results include contributions from particles in the range of 1$64 \mu \mathrm{m}$. Moreover, the PMS probe measurements of total ice crystal number densities are probably not very reliable due to the large uncertainty in the smallest size bin. The PMS probe probably underestimates the number of small particles, which would reduce the discrepancy between the predicted number density and the actual contrail properties.

\section{Summary and conclusions}

Results of three-dimensional numerical calculations of the evolution of contrails for a variety of conditions have been presented, using the large-eddy simulations (LES) technique. The model takes into account threedimensional circulations in a domain of size $640 \times 640$ $\times 640 \mathrm{~m}^{3}$ and includes most of the physical processes occurring in ice clouds in the absence of solar radiation. Apart from the aspects of the turbulence closure, it takes into account infrared radiative cooling in cloudy conditions, and forcing on large scales is considered through a prescribed vertically sheared environment. Moreover, we explicitly resolve the size distribution of ice particles and calculate the evolution of the size distributions, including processes such as deposition growth, transport, and gravitational settling.

The model has been applied to conditions that are typical for those under which persistent contrails could be observed, that is, in an atmosphere supersaturated with respect to ice and at a temperature of approximately $230 \mathrm{~K}$ or colder. The simulations start in the late dispersion phase and trace the evolution of the contrails for a half-hour duration. We have tested the sensitivity of the model to physical processes and environmental conditions and have compared the outputs of the model with measurements. Although the model includes a detailed representation of air motions, the treatment of radiative transfer and cloud microphysics is rather simplified. A major shortcoming of this model results from the neglect of the aggregation process. As a matter of fact, aggregation could probably constitute an important sink for small particles and would establish a significant growth process for large crystals in our model. Therefore, a likely consequence of omission of aggregation is that particle concentrations are overpredicted by the model. However, since no measurements of the ice crystal sticking efficiency at temperatures below about $-20^{\circ} \mathrm{C}$ are available, the potential importance of the aggregation process remains an open question. Moreover, as already outlined in section 3, a major criticism of the present simulations is that the initial conditions chosen for the simulations are too idealized so that the dynamics of the contrail evolution considered here is 
more appropriate for the time period beginning $10^{3} \mathrm{~s}$ after an aircraft has passed. Therefore, we propose that our simulations should be considered not as a faithful representation of the early timescale of a contrail, but should be viewed more as a search for basic mechanisms that tend to influence the contrail evolution. Such sensitivity tests are, in fact, one of the principal objectives of numerical models and make those a powerful tool to provide insight into the underlying dynamics.

Keeping these caveats in mind, the following major results have been obtained from the model simulations described in this paper.

- Long-lived contrails cannot be explained by the amount of water emitted by the aircraft. Although we have not performed a simulation in an atmosphere that is subsaturated with respect to ice, it is quite obvious (in comparing results from runs 3 and 8) that persistent contrails can only form in an atmosphere that is supersaturated with respect to ice.

- The flow field within the developing contrails is composed of several coherent eddies originating at the edges of the contrail. These vortices entrain moist ambient air into the contrail, which is subsequently processed into ice within the cloud. Throughout the contrail broadening process the flow field exhibits an increasingly complex structure that is due to the developing multiple vortex structure as a result of the continual production of vorticity at the cloud boundary. The maximum amplitude of vertical velocity perturbation is of the order of $0.1 \mathrm{~m} \mathrm{~s}^{-1}$ and is found at the upper edges of the contrail. The discussion on the mechanism by which the contrails obtain energy has clarified the relative importance of the various physical processes in the maintenance of contrails. We found that the turbulent kinetic energy of the contrail is produced by buoyancy. In particular, latent heating due to vapor deposition provides the significant energy source while destabilization due to differential heating plays no role owing to the small optical depth of the contrails. However, realizing the low turbulent kinetic energy levels that we found in our results, we conclude that even the energy source due to latent energy release is in fact only of minor importance in contrail evolution during the early dispersion phase, because the intensity of turbulence created by the initial downward trailing vortex pair is expected to be an order of magnitude larger than the energy levels arising in our simulations.

- Contrail evolution is controled primarily by temperature, humidity, and static stability of the ambient air. It turns out that increasing the relative humidity or warming the ambient atmosphere, respectively, will produce more vigorous eddies in the contrail. The physical reason for the enhanced energy levels in both cases is that the higher the relative humidity and the temperature of the ambient air, respectively, the greater is the conditionally available potential energy that could be converted into kinetic energy. On the other hand, reducing the relative humidity or cooling the ambient atmosphere, respectively, produces less vigorous eddies. In both cases the conversion rate from water vapor to ice, and hence the latent heat release, is much less than in the reference case owing to the initial decrease of total water content. The effect of stratification is as follows: In a neutrally stratified atmosphere enhanced energy levels are observed because the eddies have to perform no work against buoyancy forces. On the other hand, in a more stable stratified atmosphere, stable stratification counteracts vertical motions so that mixing is much slower in the vertical than in the horizontal direction.

- Vertical shear of the environmental flow is of minor importance in the evolution of contrails at least during the 30-min simulation interval. Admittedly, our model experiments may have only restricted applicability to the question of the potential impact of vertical wind shear since the assumed shear cases enclose only very weak shear. Moreover, if a larger vertical extent for the initial contrail had been chosen, the effects of vertical wind shear would probably be nonnegligible, even at the low levels considered. We recommend that this effect be explored further with other models.

- The effect of radiative transfer on the particle condensational growth and on thermodynamic processes leads to insignificant changes in the structure of the modeled contrail. This is due to the small particle sizes and small cooling rates that we found in the contrail.

- Small differences in the characterization of the initial particle size have noticeable but little effect on the properties of the modeled contrail; a smaller initial effective radius produces higher turbulent kinetic energy levels and increased cloud ice contents.

- Comparison of our results with aircraft microphysical measurements made over the North Sea indicates that the simulations generate reasonable ice content, but the predicted ice number concentrations appear too high. The discrepancies between our simulations and the ICE data can be attributed to a combination of errors due to missing physical processes that may be important, lack of information about initial and environmental conditions, and uncertainties about experimental estimates of microphysical parameters.

Beyond a doubt, a more detailed comparison of results to observations would be desirable. However, a more definite assessment of the realism of the model simulation awaits the acquisition of an appropriate set of comprehensive observations that could be used to verify the model predictions and to see whether the essential processes are included in the model. Therefore, we call for key measurements that would assist in addressing the contrail issue. Such kind of observations should include multiple, high-quality upper tropospheric humidity, turbulence, and microphysical measurements in the same contrail at various ages. In the future we 
intend to apply this model to further contrail observations when the required datasets become available.

Certainly, the simulations presented are far from being perfect, and they did not treat all aspects of contrail evolution. This drawback may be overcome in the future by a closer coupling of the present simulations to the earlier phases of contrail evolution. This could be achieved by initializing the dynamic and thermodynamic fields utilizing the output of a jet-vortex-phase-resolving model (e.g., that of Gerz and Ehret 1996). Moreover, in order to get more adequate results for the whole dispersion phase the effect of ambient background turbulence on contrail evolution should be addressed in future studies. We hope that studies with this model will contribute to the development of physically well-based contrail parameterization for use in general circulation models to address the climatic impact of contrail-induced cirrus clouds.

Acknowledgments. The author wishes to thank Drs. H. Grassl and H. Hinzpeter for their useful comments and close interest in this study. Thanks also to Mrs. B. Zinecker for typing the manuscript. This work has received financial support by the German Bundesministerium für Bildung, Wissenschaft, Forschung und Technologie $(\mathrm{BMBF})$ within the project Schadstoffe in der Luftfahrt.

\section{REFERENCES}

Ackerman, A. S., O. B. Toon, and P. V. Hobbs, 1995: A model for particle microphysics, turbulent mixing, and radiative transfer in the stratocumulus-topped marine boundary layer and comparison with measurements. J. Atmos. Sci., 52, 1204-1236.

Appleman, H., 1953: The formation of exhaust condensation trails by jet aircraft. Bull. Amer. Meteor. Soc., 34, 14-20.

Arnason, G., and P. S. Brown, 1971: Growth of cloud droplets by condensation: A problem in computational stability. J. Atmos. Sci., 28, 72-77.

Bakan, S., M. Betancor, V. Gayler, and H. Graß1, 1994: Contrail frequency over Europe from NOAA-satellite images. Ann. Geophys., 12, 962-968.

Baumann, R., R. Busen, H. P. Fimpel, C. Kiemle, M. E. Reinhardt, and M. Quante, 1993: Measurements of contrails of commercial aircraft. Preprints, Proc. Eighth Symp. on Meteorological Observations and Instrumentation, Anaheim, CA, Amer. Meteor. Soc., 484-489.

Beard, K. V., 1976: Terminal velocity and shape of cloud and precipitation drops aloft. J. Atmos. Sci., 33, 851-864.

Berry, E. X., 1967: Cloud droplet growth by collection. J. Atmos. Sci., 24, 688-701.

Boin, M., and L. Levkov, 1994a: Numerical simulation of the life time of contrails. Impact of Emissions from Aircraft and Spacecraft upon the Atmosphere, U. Schumann and D. Wurzel, Eds., DLR-Mitteilung, 430-435.

$\longrightarrow$, and - , 1994b: A numerical study of contrail development. Ann. Geophys., 12, 969-978.

Bott, A., U. Sievers, and W. Zdunkowski, 1990: A radiation fog model with a detailed treatment of the interaction between radiative transfer and fog microphysics. J. Atmos. Sci., 47, 2153-2166.

Chlond, A., 1992: Three-dimensional simulation of cloud street development during a cold air outbreak. Bound.-Layer Meteor., 58, 161-200.
- 1994 : Locally modified version of Bott's advection scheme. Mon. Wea. Rev., 122, 111-125.

CIAP, 1975: Climatic Impact Assessment Program. Final Report DOT-TST-75-51 (8 vols.), 765 pp. [Available from the National Technical Information Service, Springfield, VA 22151.]

Cox, S. K., 1976: Observations of cloud infrared effective emissivity. J. Atmos. Sci., 33, 287-289.

Deardorff, J. W., 1980: Stratocumulus-capped mixed layers derived from a three-dimensional model. Bound.-Layer Meteor., 18, 495-527.

Detwiler, A., and R. Pratt, 1984: Clear-air seeding: Opportunities and strategies. J. Wea. Mod., 16, 46-60.

Dürbeck, T., and T. Gerz, 1995: Large-eddy simulation of aircraft exhaust plumes in the free atmosphere: effective diffusivities and cross-sections. Geophys. Res. Lett., 22, 3203-3206.

Fortuin, J. P. F., R. van Dorland, W. M. F. Wauben, and H. Kelder, 1995: Greenhouse effects of aircraft emissions as calculated by a radiative transfer model. Ann. Geophys., 13, 413-418.

Freudenthaler, V., F. Homburg, and H. Jäger, 1995: Contrail observations by ground-based scanning lidar: Cross-sectional growth. Geophys. Res. Lett., 22, 3501-3504.

Gage, K. S., 1979: Evidence for a $k^{-5 / 3}$ law inertial range in mesoscale two-dimensional turbulence. J. Atmos. Sci., 36, 1950-1954.

Gayet, J.-F., G. Febvre, G. Brogniez, H. Chepper, W. Renger, and P. Wendling, 1996: Microphysical and optical properties of cirrus and contrails: Cloud field study on 13 October 1989. J. Atmos. Sci., 53, 126-138.

Gerz, T., and U. Schumann, 1991: Direct simulation of homogeneous turbulence and gravity waves in sheared and unsheared stratified flows. Turbulent Shear Flow 7, F. Durst et al., Eds., SpringerVerlag, 27-45.

, and T. Ehret, 1996: Wake dynamics and exhaust distribution behind cruising aircraft. DLR-Report No. 58, 12 pp. [Available from Institut für Physik der Atmosphäre DLR, Oberpfaffenhofen, D-82230 Wessling, Germany.]

Gierens, K. M., 1994: The influence of radiation on the diffusional growth of ice crystals. Contrib. Atmos. Phys., 67, 181-193.

— 1996 : Numerical simulations of persistent contrails. J. Atmos. Sci., 53, 3333-3348.

Grassl, H., 1990: Possible climatic effects of contrails and additional water vapour. Air-Traffic and the Environment, U. Schumann, Ed., Springer-Verlag, 124-137.

Greene, G. C., 1986: An approximate model of vortex decay in the atmosphere. J. Aircraft, 23, 566-573.

Hall, W. D., 1980: A detailed microphysical model within a twodimensional dynamic framework: Model description and preliminary results. J. Atmos. Sci., 37, 2486-2507.

- and H. R. Pruppacher, 1976: The survival of ice particles falling from cirrus clouds in subsaturated air. J. Atmos. Sci., 33, 19952006.

Hobbs, P. V., S. Chang, and J. S. Locatelli, 1974: The dimensions and aggregation of ice crystals in natural clouds. J. Geophys. Res., 79, 2199-2206.

Jensen, E. J., O. B. Toon, L. Pfister, and H. B. Selkirk, 1996: Dehydration of the upper troposphere and lower stratosphere by subvisible cirrus clouds near the tropical tropopause. Geophys. Res. Lett., 23, 825-828.

Johnson, C., J. Henshaw, and G. McInnes, 1992: Impact of aircraft and surface emissions of nitrogen oxides on tropospheric ozone and global warming. Nature, 355, 69-71.

Kajikawa, M., and A. J. Heymsfield, 1989: Aggregation of ice crystals in cirrus. J. Atmos. Sci., 46, 3108-3121.

Knollenberg, R. G., 1972: Measurements of the growth of the ice budget in a persisting contrail. J. Atmos. Sci., 29, 1367-1374.

Laube, M., and H. Höller, 1988: Cloud physics. Meteorology, Vol. 4b, Physical and Chemical Properties of the Air, G. Fischer, Ed., Springer-Verlag, 1-100.

Lewellen, D. C., and W.-S. Lewellen, 1996: Large-eddy simulations of the vortex-pair breakup in aircraft wakes. AIAA J., 34, 23372345 . 
Lilly, D. K., 1983: Stratified turbulence and mesoscale variability of the atmosphere. J. Atmos. Sci., 40, 749-761.

_, 1988: Cirrus outflow dynamics. J. Atmos. Sci., 45, 1594-1605.

Liou, K. N., 1992: Radiation and Cloud Processes in the Atmosphere. Oxford University Press, $487 \mathrm{pp}$.

Louisnard, N., C. Baudoin, G. Billet, F. Garnier, D. Hills, T. Mentel, P. Mirabel, J. C. Petit, J. L. Schultz, D. Tabeb, J. Thlibi, A. Wahner, and P. Woods, 1995: Physics and chemistry in the aircraft wake. The Impact of $\mathrm{NO}_{x}$ Emissions from Aircraft upon the Atmosphere at Flight Altitudes 8-15 km (AERONOX), U. Schumann, Ed., EC-DLR Publications, 195-309.

Mitchell, D. L., 1988: Evolution of snow size spectra in cyclonic storms. Part I: Snow growth by vapor deposition and aggregation. J. Atmos. Sci., 45, 3431-3451.

- 1991: Evolution of snow size spectral in cyclonic storms. Part II: Deviations from the exponential form. J. Atmos. Sci., 48, 1885-1899.

_ 1994: A model predicting the evolution of ice particle size spectra and radiative properties of cirrus clouds. Part I: Microphysics. J. Atmos. Sci., 51, 797-816.

Monin, A. S., and A. M. Yaglom, 1971: Statistical Fluid Mechanics: Mechanics of Turbulence. The MIT Press, 769 pp.

Piacsek, S. A., and G. P. Williams, 1970: Conservation properties of convection difference schemes. J. Comput. Phys., 6, 392-405.

Ponater, M., S. Brinkop, R. Sausen, and U. Schumann, 1996: Simulating the global atmospheric response to aircraft water vapor emissions and contrails: A first approach using a GCM. Ann. Geophys., 14, 941-960.

Prabhakara, C., D. P. Kratz, J.-M. Yoo, G. Dalu, and A. Vernekar, 1993: Optically thin cirrus clouds: Radiative impact on the warm pool. J. Quant. Spectrosc. Radiat. Transfer, 49, 467-483.

Pratt, R. W., 1985: Review of radiosonde humidity and temperature errors. J. Atmos. Oceanic Technol., 2, 404-407.

Sassen, K., 1991: Aircraft-produced ice particles in a highly supercooled altocumulus cloud. J. Appl. Meteor., 30, 765-775.

- M. Griffin, and G. C. Dodd, 1989: Optical scattering and microphysical properties of subvisual cirrus clouds and climatic implications. J. Appl. Meteor., 28, 91-98.

Schilling, V., S. Siano, and D. Etling, 1996: Dispersion of aircraft emissions due to wake vortices in stratified shear flows: A twodimensional numerical study. J. Geophys. Res., 101, 2096520974.

Schumann, U., 1994: On the effect of emissions from aircraft engines on the state of the atmosphere. Ann. Geophys., 12, 365-384.

- 1995: The impact of $\mathrm{NO}_{\mathrm{x}}$ emissions from aircraft upon the atmosphere at flight altitudes $8-15 \mathrm{~km}$ (AERONOX). CEC Report EUR $16209 \mathrm{EN}, 471 \mathrm{pp}$. [Available from Office for Publications of the EC DG XII, Rue de la Loi 200, B-1049 Brussels, Belgium.]

_ 1996 : On conditions for contrail formation from aircraft exhausts. Meteor. Z., N. F. 5, 4-23.

_ , and P. Wendling, 1990: Determination of contrails from satellite data and observational results. Air Traffic and the Environment, U. Schumann, Ed., Springer-Verlag, 138-153.

- , and T. Gerz, 1995: Turbulent mixing in stably stratified shear flows. J. Appl. Meteor., 34, 33-48.

- , P. Konopka, R. Baumann, R. Busen, T. Gerz, H. Schlager, P. Schulte, and H. Volkert, 1995: Estimate of diffusion parameters of aircraft exhaust plumes near the tropopause from nitric oxide and turbulence measurements. J. Geophys. Res., 100, $14147-$ 14162.

Sommeria, G., and J. W. Deardorff, 1977: Subgrid-scale condensation in models of nonprecipitating clouds. J. Atmos. Sci., 34, 344355.

Starr, D. O'C., and S. K. Cox, 1985: Cirrus clouds. Part I: A cirrus cloud model. J. Atmos. Sci., 42, 2663-2681.

Stephens, G. L., and P. J. Webster, 1981: Clouds and climate: Sensitivity of simple systems. J. Atmos. Sci., 38, 235-247.

— S.-C. Tsay, P. W. Stackhouse, and P. J. Flatau, 1990: The relevance of the microphysical and radiative properties of cirrus clouds to climate and climate feedback. J. Atmos. Sci., 47, 17421753.

Tripoli, G. J., and W. R. Cotton, 1981: The use of ice-liquid water potential temperature as a thermodynamic variable in deep atmospheric models. Mon. Wea. Rev., 109, 1094-1102.

Young, K. C., 1974: A numerical solution of wintertime, orographic precipitation: Part I. Description of model microphysics and numerical techniques. J. Atmos. Sci., 31, 1735-1748. 